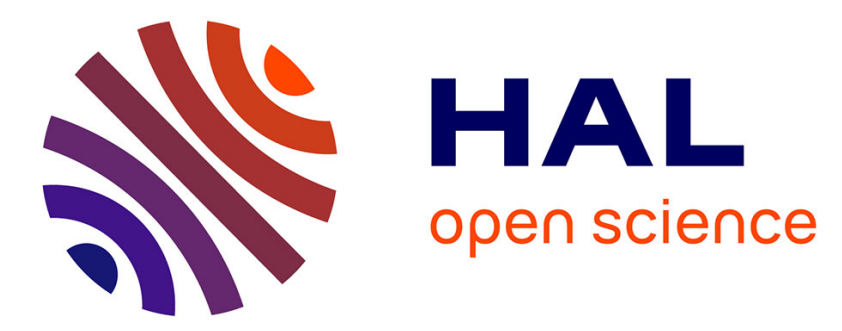

\title{
Input-output measures for model reduction and closed-loop control: Application to global modes
}

Alexandre Barbagallo, Denis Sipp, Peter J. Schmid

\section{To cite this version:}

Alexandre Barbagallo, Denis Sipp, Peter J. Schmid. Input-output measures for model reduction and closed-loop control: Application to global modes. Journal of Fluid Mechanics, 2011, 685, pp.23-53. 10.1017/jfm.2011.271 . hal-00997981

HAL Id: hal-00997981

https://hal-polytechnique.archives-ouvertes.fr/hal-00997981

Submitted on 5 Jun 2014

HAL is a multi-disciplinary open access archive for the deposit and dissemination of scientific research documents, whether they are published or not. The documents may come from teaching and research institutions in France or abroad, or from public or private research centers.
L'archive ouverte pluridisciplinaire HAL, est destinée au dépôt et à la diffusion de documents scientifiques de niveau recherche, publiés ou non, émanant des établissements d'enseignement et de recherche français ou étrangers, des laboratoires publics ou privés. 


\title{
Input-output measures for model reduction and closed-loop control: application to global modes
}

\author{
Alexandre Barbagallo ${ }^{1,2}$, Denis Sipp ${ }^{1}$ \\ and Peter J. Schmid ${ }^{2} \dagger$ \\ ${ }^{1}$ ONERA-DAFE, 8 rue des Vertugadins, 92190 Meudon, France \\ ${ }^{2}$ Laboratoire d'Hydrodynamique (LadHyX), CNRS-Ecole Polytechnique, 91128 Palaiseau, France
}

(Received 26 August 2010; revised 1 April 2011; accepted 15 June 2011)

Feedback control applications for flows with a large number of degrees of freedom require the reduction of the full flow model to a system with significantly fewer degrees of freedom. This model-reduction process is accomplished by Galerkin projections using a reduction basis composed of modal structures that ideally preserve the input-output behaviour between actuators and sensors and ultimately result in a stabilized compensated system. In this study, global modes are critically assessed as to their suitability as a reduction basis, and the globally unstable, two-dimensional flow over an open cavity is used as a test case. Four criteria are introduced to select from the global spectrum the modes that are included in the reduction basis. Based on these criteria, four reduced-order models are tested by computing open-loop (transfer function) and closed-loop (stability) characteristics. Even though weak global instabilities can be suppressed, the concept of reduced-order compensators based on global modes does not demonstrate sufficient robustness to be recommended as a suitable choice for model reduction in feedback control applications. The investigation also reveals a compelling link between frequency-restricted input-output measures of open-loop behaviour and closed-loop performance, which suggests the departure from mathematically motivated $\mathscr{H}_{\infty}$-measures for model reduction toward more physically based norms; a particular frequency-restricted input-output measure is proposed in this study which more accurately predicts the closed-loop behaviour of the reduced-order model and yields a stable compensated system with a markedly reduced number of degrees of freedom.

Key words: control theory, instability control

\section{Introduction}

As active control strategies are increasingly applied to high-dimensional and complex flow configurations arising in industrial and academic settings, model reduction becomes a key technology. The goal of model reduction is the projection of the high degree-of-freedom flow dynamics onto a smaller system which is subsequently used in estimating the flow state based on measurement information. There is a considerable amount of choice for the bases of this projection which in the

$†$ Email address for correspondence: peter@ladhyx.polytechnique.fr 
past yielded a wide range of reduction strategies but also varied results. In this article we specifically consider global modes for the reduction basis, develop measures and tools to assess their effectiveness and comment on their suitability for feedback control applications based on reduced-order models.

In our study we will concentrate on two-dimensional incompressible flow over an open square cavity at supercritical Reynolds numbers, a flow configuration that exhibits oscillator behaviour in the form of a self-sustained cyclic perturbation dynamics which is rather insensitive to stochastic outside influences. A global stability analysis of this type of flow exhibits unstable global modes and provides frequencies and growth rates as well as the spatial shapes of the self-sustained structures. In many industrial applications, oscillatory behaviour is typical and often a source of acoustic radiation and/or structural excitation which can cause material fatigue or damage. For this reason, passive and active control strategies to weaken or suppress these oscillations are of great interest.

Passive control strategies, such as mean flow modifications, do not add energy to the system and are particularly attractive due to their simplicity of implementation and efficiency (see Strykovski \& Sreenivasan 1990; Giannetti \& Luchini 2007; Marquet, Sipp \& Jacquin 2008), even though a non-negligible drag penalty may occur. However, while these strategies can successfully extend the parameter range of stable flows, they ultimately may not be able to prevent the onset of instabilities as a critical parameter value is exceeded. In this case, due to their time-independent design, they can no longer influence the unsteady nature of the flow. Active control, on the other hand, adds energy to the system in the form of a predesigned actuation (open-loop control) or an actuation using flow information from sensor measurements (closed-loop control). Among the closed-loop control theories, the linear quadratic Gaussian (LQG) strategy (see Burl 1999; Zhou, Doyle \& Glover 2002) has been considered for and successfully applied to the feedback control of unsteady flows (see e.g. Bewley \& Liu 1998; Kim \& Bewley 2007; Bagheri, Brandt \& Henningson 2009a; Barbagallo, Sipp \& Schmid 2009; Sipp et al. 2010). This method is based on a linear description of the flow behaviour and includes an estimator that optimally reconstructs an approximate flow field from sensor measurements. This approximate flow state provides the basis on which an optimal control law is designed. The optimization of the estimation and control leads to two Riccati equations which can only be solved for a rather low number of degrees of freedom. It is thus desirable and paramount to reduce the size of the compensator (i.e. the combined estimator and controller) using a procedure known as model reduction. However, since this reduction procedure implies a loss of information, it is of critical importance to retain only the relevant features of the original model in the reduced-order model. The key feature to be conserved is the input-output behaviour, i.e. the effect of the actuator on the sensor; its conservation is a sufficient condition for the success of LQG control design based on reduced-order models.

Among the various procedures to obtain a reduced-order model, the Galerkin projection method has been widely used in flow control applications. This method consists of projecting the Navier-Stokes equations (or linearized Navier-Stokes equations) onto spatial modal structures (Antoulas, Sorensen \& Gugercin 2001; Antoulas 2005). The choice of these modes is pivotal and changes considerably the properties of the resulting reduced-order model (see Bagheri et al. 2009b; Barbagallo et al. 2009). A common choice is to use proper orthogonal decomposition (POD) modes as the projection basis. These modes are optimal in describing the most energetic structures of the flow (Lumley 1970; Sirovich 1987; Berkooz, Holmes \& 
Lumley 1993) but yield reduced-order models which are not optimal for closed-loop control applications since they do not take into account the flow's observability by the sensors. This difficulty is overcome by projecting onto balanced proper orthogonal decomposition (BPOD) modes which, by construction, give equal emphasis to the controllability and observability of the flow (Moore 1981; Rowley 2005). Closed-loop control with reduced-order compensators based on POD and BPOD modes have been studied on various globally unstable configurations (see Barbagallo et al. 2009; Ahuja \& Rowley 2010) where stabilization of the flow could be achieved within the limitations of the linear approximation.

Reduced-order models obtained by a Galerkin projection onto global modes have also been considered in previous work in an attempt to control globally unstable flows. The flow over a shallow cavity has been successfully stabilized in Åkervik et al. (2007) and Henningson \& Åkervik (2008) using a reduced-order model merely based on the unstable and a few stable global modes. A similar model, however, failed to suppress the instabilities over an open square cavity, irrespective of the number of stable global modes added to the reduced-order model (Barbagallo et al. 2009). This failure has been attributed to the ineffectiveness of global modes in capturing the input-output behaviour of the original system which could further be linked to the strong non-normality of the global modes yielding, in turn, extreme controllability coefficients. This feature has subsequently been observed by Ehrenstein, Passaggia \& Gallaire (2010) in a study of the separated flow over a shallow bump. Introducing the so-called double-projection (i.e. an orthogonal projection for the actuator combined with a bi-orthogonal projection for the remaining terms in the governing equations) in an attempt to reduce in magnitude the coefficients arising from the projection of the actuator, Ehrenstein et al. (2010) were able to stabilize the separated flow.

In the face of these diverse and possibly conflicting results, it seems helpful and beneficial to assess and clarify the suitability of global modes as a reduction basis in closed-loop control design for globally unstable flows. This is the primary aim of this article. A second, and even more important, objective is the development of appropriate input-output measures that allow the most efficient design of reducedorder models. For example, balanced truncation, one of the most effective techniques for model reduction in feedback control applications, minimizes the $\mathscr{H}_{\infty}$-error between the full and reduced transfer function (see e.g. Antoulas 2005); yet, it may appear too stringent and limiting to focus on the worst-case error over all frequencies and to tacitly neglect the flow response contained in other frequencies. Our present studies suggest frequency-restricted input-output measures that reflect physical attributes of the underlying fluid dynamics which in turn result in more effective reduced-order models. We will show that concentrating on relevant frequency bands (given by the unstable global modes) one can bring the size of the reduced-order model below the one given by the $\mathscr{H}_{\infty}$-design without compromising the stability of the compensated system.

We will begin with a brief description of the flow configuration $(\S 2)$ together with the mathematical formulation of our problem. After a short outline of closed-loop control and model reduction techniques $(\S 3)$, we will introduce and motivate selection criteria for the inclusion of global modes in a reduced-order model $(\S 4)$. Openloop transfer functions and stability properties of the compensated system will aid in assessing the effectiveness of the criteria and, more generally, the limitations of reduced-order models based on global modes $(\S 5)$. A representative case $(R e=7500)$ will be treated in detail. In particular, the introduction of frequency-limited norms for the transfer function error will show that further significant reductions in the size 


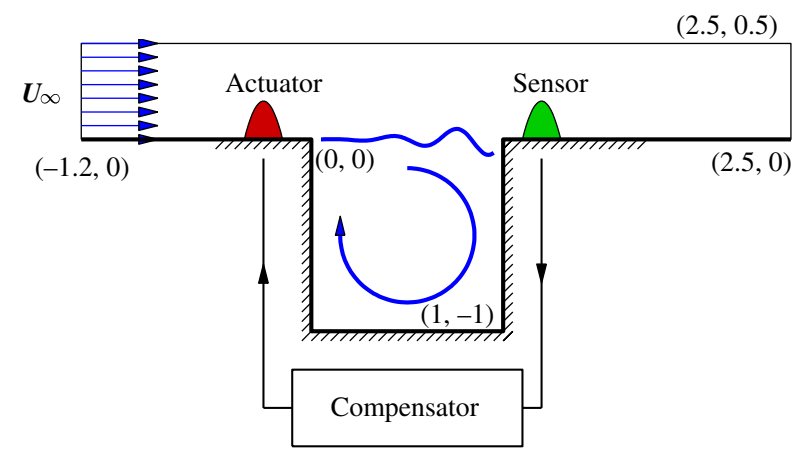

FIGURE 1. (Colour online available at journals.cambridge.org/flm) Sketch of flow over an open cavity.

of the reduced-order model are possible. Key properties of the analysis will then be investigated for a range of Reynolds numbers (\$6). A summary and concluding remarks will complete this article.

\section{Flow configuration and governing equations}

The flow configuration chosen to address the suitability of global modes for closed-loop control applications is the two-dimensional incompressible flow over an open square cavity. The geometry and control set-up is shown in figure 1 and is identical to the one studied in Barbagallo et al. (2009). The flow enters the domain from the left with a uniform horizontal velocity $\boldsymbol{U}_{\infty}$ after which a boundary layer develops. It then detaches at the left side of the cavity and forms a shear layer instability if the critical Reynolds number (based on the incoming velocity and the length/depth $D$ of the cavity) exceeds $R e_{c}=4140$ (see Sipp \& Lebedev 2007). The shear layer separates the recirculation motion inside the cavity from the external flow. Unstable flows that are characterized by self-sustained oscillatory motion, like the open cavity flow at supercritical Reynolds numbers, are often referred to as oscillators within a global stability framework. In this present study, we will use active feedback control schemes and attempt to stabilize the flow for marginal and supercritical Reynolds numbers ranging from $R e=4140$ to $R e=7500$.

The essential mechanism for the development of a global instability is based on a combination of the linear amplification of specific infinitesimal perturbations followed by their saturation due to nonlinear effects once finite amplitudes have been reached. In the present approach we aim to stabilize the flow in its linear regime, i.e. under conditions when the small perturbation amplitudes justify the use of a linearized model of the flow. This model consists of the Navier-Stokes equations linearized about a base flow $\mathbf{u}_{0}=\left(u_{0}, v_{0}\right)$. This base flow, which represents a solution of the nonlinear Navier-Stokes equations, is unstable to perturbations and may be computed using a Newton-Raphson technique. The linearized evolution equations for the perturbation field $\mathbf{u}=(u, v, p)$ are then derived and non-dimensionalized using the length of the cavity $D$ and the incoming uniform velocity $\boldsymbol{U}_{\infty}$. They are

$$
\begin{gathered}
\frac{\partial \mathbf{u}}{\partial t}+(\mathbf{u} \cdot \nabla) \mathbf{u}_{0}+\left(\mathbf{u}_{0} \cdot \nabla\right) \mathbf{u}=-\nabla p+\frac{1}{R e} \Delta \mathbf{u}, \\
\nabla \cdot \mathbf{u}=0,
\end{gathered}
$$


where $\nabla$ (resp. $\Delta$ ) is the gradient (resp. the Laplacian) operator. The above equations are spatially discretized using finite elements of Taylor-Hood type (P2-P2-P1) implemented in the software Freefem ++ (Hecht et al. 2005). The discretized system of equations can formally be written in matrix form as

$$
Q \frac{\mathrm{d} \boldsymbol{X}}{\mathrm{d} t}=A \boldsymbol{X},
$$

where $\boldsymbol{X}=(U V P)^{\mathrm{T}}$ is the state vector composed of the streamwise velocity $U$, the normal velocity $V$ and the pressure $P$. The matrix $A$ represents the linearized Navier-Stokes operator while the matrix $Q$ contains the weights arising from the discretization by finite elements. For our case, the total number of degrees of freedom of the discretized system (i.e. the size of the matrices $Q$ and $A$ ) is approximately $n=900000$. The kinetic energy of the flow can be defined according to

$$
\|\boldsymbol{X}\|_{2}^{2}=\langle\boldsymbol{X}, \boldsymbol{X}\rangle=\boldsymbol{X}^{*} Q \boldsymbol{X},
$$

where * denotes the transpose conjugate operator. The actuation on the flow by the controller is modelled as a Gaussian body force on the vertical velocity. This forcing is spatially localized near the upstream edge of the cavity where it has a distinct effect on the shear layer and, consequently, on the instability mechanism. Approximating a realistic actuator requires its spatial extent to be small compared to the size of the cavity; we choose

$$
v(x, y)=\exp \left[-\frac{\left(x-x_{0}\right)^{2}+\left(y-y_{0}\right)^{2}}{2 \sigma^{2}}\right]
$$

with $x_{0}=-0.1, y_{0}=0.02$ and $\sigma=0.0849$. These specific values define a Gaussian function of unit amplitude and width 0.2. Using a body force (instead of a lifting procedure) has the advantage of a direct comparison with previous work (Åkervik et al. 2007; Ehrenstein et al. 2010) and results in a simplified formulation of the control problem compared to a true actuation at the wall (see Barbagallo et al. 2009). The full control effort is obtained by multiplying the spatial Gaussian profile by a temporal scalar function $u(t)$ which transforms (2.2) into a forced problem

$$
Q \frac{\mathrm{d} \boldsymbol{X}}{\mathrm{d} t}=A \boldsymbol{X}+Q \boldsymbol{B} u,
$$

where $\boldsymbol{B}$ is a real vector of dimension $n$ arising from the spatial discretization of the actuator using the finite-element bases.

Within a closed-loop (feedback) framework the temporal control law $u(t)$ is to be computed in real time using real-time measurements of the system. In the present work we choose to measure the wall-shear stress at the downstream edge of the cavity, expressed mathematically as

$$
m(t)=\left.\int_{x=1}^{x=1.1} \frac{\partial u}{\partial y}\right|_{y=0} \mathrm{~d} x,
$$

which, after finite-element discretization, yields $m=\boldsymbol{C} \boldsymbol{X}$ with $\boldsymbol{C}$ as a real column vector of length $n$ extracting the wall-shear stress from the full state vector $\boldsymbol{X}$. This concludes the state-space formulation of the flow control problem given by

$$
\begin{gathered}
Q \frac{\mathrm{d} \boldsymbol{X}}{\mathrm{d} t}=\boldsymbol{A} \boldsymbol{X}+Q \boldsymbol{B} u, \\
m=\boldsymbol{C} \boldsymbol{X} .
\end{gathered}
$$




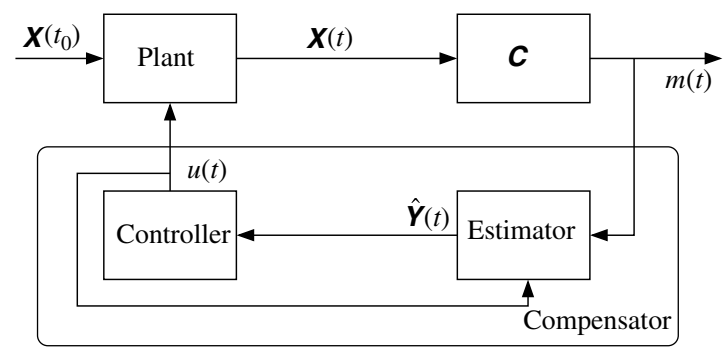

FIGURE 2. Block diagram of feedback control set-up showing the plant, estimator and controller components.

In what follows, this system of equations will form the basis for control design and model reduction efforts.

\section{Closed-loop control design and model reduction}

At Reynolds numbers above the critical one, instabilities arise that ultimately lead to exponential growth in kinetic perturbation energy within the linear framework. In order to suppress these instabilities we consider an active closed-loop control strategy that exploits real-time flow information from the sensor and manipulates the flow via the actuator such that a user-specified objective (the suppression of instabilities) is achieved with optimal effort.

\subsection{Linear quadratic Gaussian ( $L Q G)$ control}

The design of such a control strategy takes advantage of the widely used LQG theory (Burl 1999; Zhou et al. 2002). The underlying principle of this theory is graphically presented in figure 2. In this sketch the plant represents the inherent (uncontrolled) dynamics of the flow, in our case governed by (2.5). The output from the sensor, i.e. the measurement $m(t)=\boldsymbol{C} \boldsymbol{X}$, represents the sole information on which the control law will be based. This measurement is then fed into an estimator which recovers an approximate state $\hat{\boldsymbol{Y}}$ which is subsequently multiplied by the control gain $\hat{K}$ to produce a control signal $u(t)$; this signal is finally fed back into the flow system. The objective of the control is the minimization of a given cost functional, in our case, the kinetic energy of the perturbation. In addition, we consider the small-gain limit where control efforts are strongly penalized; in this limit, successful control results in the reflection of the unstable eigenvalues across the neutral line once the compensator is applied. The design of both an estimator - in particular, the Kalman gain $\hat{L}$ which optimally exploits measurement information to recover state information - and a controller requires the solution of algebraic Riccati equations, a matrix equation that can only be solved by direct techniques for a rather limited number of degrees of freedom. For this reason, the feedback loop from measurement $m(t)$ to control $u(t)$ via the estimator and controller has to be based on a substitute system of significantly fewer degrees of freedom, i.e. on a reduced-order model (ROM). With this reduced-order model in place, the Riccati equations for the control gain $\hat{K}$ and Kalman gain $\hat{L}$ can be solved by standard techniques (e.g. Datta 2003).

\subsection{Model reduction basics}

The goal of model reduction is to represent pertinent features of the original system (2.7) with a reduced number of states. In its most common form this reduction 
is accomplished by a Petrov-Galerkin projection of the full system onto an identified basis. The choice of this basis then determines the effectiveness and accuracy of the reduced system. In the present study, we will evaluate the performance of reducedorder models obtained from a projection onto global modes and assess their efficiency when incorporated into a feedback control loop. To this end, we gather a selection of $n_{r}$ global modes as columns of the $n \times n_{r}$ matrix $V$. The details of the selection will be left undetermined at this point, but will be specified later. The state $\boldsymbol{X}$ will then be expressed in this basis as

$$
\boldsymbol{X}(t)=V \hat{\boldsymbol{X}}(t),
$$

where $\hat{\boldsymbol{X}}$ is a column vector of length $n_{r}$, denoting the reduced state (the symbol $\hat{.}$ will be used to indicate reduced variables or matrices). The $i$ th component of $\hat{\boldsymbol{X}}$ represents the dynamics of the corresponding $i$ th global mode $V_{(:, i)}$.

Defining a second basis $W$ that is bi-orthogonal to $V$ according to $W^{*} Q V=I$, the reduced state variable $\hat{\boldsymbol{X}}$ is given by

$$
\hat{X}=W^{*} Q X .
$$

The reduced system in state-space form, obtained by applying the Petrov-Galerkin projection to (2.7), is written

$$
\begin{gathered}
\frac{\mathrm{d} \hat{\boldsymbol{X}}}{\mathrm{d} t}=\hat{\boldsymbol{\Lambda}} \hat{\boldsymbol{X}}+\hat{\boldsymbol{B}} u, \\
m=\hat{\boldsymbol{C}} \hat{\boldsymbol{X}},
\end{gathered}
$$

where the reduced system, control and measurement matrices are respectively given by

$$
\hat{\Lambda}=W^{*} A V, \quad \hat{\boldsymbol{B}}=W^{*} Q \boldsymbol{B}, \quad \hat{\boldsymbol{C}}=\boldsymbol{C} V .
$$

The choice of global modes as a reduction basis yields a diagonal reduced system matrix $\hat{\Lambda}$ containing the associated eigenvalues of the selected global modes.

\section{Reduced-order models based on global modes}

To study the linear stability of non-parallel two- or three-dimensional base flows a global stability analysis is commonly applied. It consists of a decomposition of the perturbation into a complex exponential time dependence and a purely spatial structure, referred to as a global mode:

$$
\boldsymbol{X}(t)=\tilde{\boldsymbol{X}} \exp (\lambda t) \quad \text { where } \lambda=\sigma+\mathrm{i} \omega .
$$

The asymptotic temporal behaviour of each global mode is then governed by its growth rate $\sigma$ and frequency $\omega$. Positive growth rates $(\sigma>0)$ indicate global instability.

\subsection{Direct and adjoint global modes}

The assumption (4.1) transforms the initial-value problem (2.2) into a generalized eigenvalue problem for the eigenvalue $\lambda$ and the global mode $\tilde{\boldsymbol{X}}$. We have

$$
A \tilde{\boldsymbol{X}}=\lambda Q \tilde{\boldsymbol{X}},
$$

which has to be solved by iterative techniques owing to the size $\left(\sim 10^{6} \times 10^{6}\right)$ of the matrices $A$ and $Q$. A shift-invert Arnoldi algorithm (see Lehoucq \& Scott 1997) has 
Reynolds number $R e$

Number of unstable modes

Number of computed stable modes $\begin{array}{llllll}5250 & 5500 & 6000 & 6500 & 7000 & 7500\end{array}$

$\begin{array}{cccccc}4 & 4 & 6 & 6 & 6 & 8 \\ 1324 & 1391 & 1511 & 1636 & 1764 & 1875\end{array}$

TABLE 1. Number of unstable and computed stable global modes for flow over an open square cavity at various Reynolds numbers considered in this study.

been used to determine the global spectrum, where we restricted our computations to growth rates above $\sigma=-4$. Details of the numerical procedure can be found in Barbagallo et al. (2009). Depending on the Reynolds number Re the number of eigenvalues found in this domain varies from 1328 to 1883 modes (see table 1). For eigensolutions with decay rates lower than $\sigma \approx-4$ first effects of round-off errors are encountered which are exacerbated until they entirely inhibit computations of global modes beyond $\sigma<-8$ due to the non-normality of the matrix $A$. Moreover, the availability of $O\left(10^{3}\right)$ global modes for a model reduction effort was deemed sufficient for the reduced-order model to still qualify as 'reduced'; ideally, many fewer modes should be necessary.

The matrix $A$ is non-normal, resulting in a set of non-orthogonal global modes (Schmid \& Henningson 2000). An additional basis $W$ - the set of adjoint global modes - is thus necessary. The adjoint global modes are solutions of the adjoint eigenvalue problem

$$
A^{*} \tilde{\boldsymbol{X}}^{+}=\lambda^{*} Q \tilde{\boldsymbol{X}}^{+}
$$

The adjoint global modes are denoted by $\tilde{\boldsymbol{X}}^{+}$; the eigenvalues of the adjoint problem are simply the complex conjugate of the direct eigenvalues $\lambda$. After proper normalization, the direct and adjoint global modes satisfy the bi-orthogonality relation

$$
\tilde{\boldsymbol{X}}_{i}^{+*} Q \tilde{\boldsymbol{X}}_{j}=\delta_{i j}
$$

with $\delta_{i j}$ the Kronecker symbol.

\subsection{Analysis of the spectrum}

Since we wish to construct reduced-order models based on global modes, it will be instructive to analyse the properties and characteristics of the eigensolutions of $A$. We will focus on the case $R e=7500$, but would like to stress that equivalent results have been obtained for lower Reynolds numbers. The global spectrum (restricted to the half-plane $\omega \geqslant 0$ due to symmetry) for $R e=7500$ is displayed in figure 3 . At this parameter setting, we have eight unstable global modes with $\sigma>0$ (four appear in the $\omega$-half-plane in figure 3). The spatial structure of the most unstable global mode is presented in figure 4 , visualized by contours of the streamwise velocity. It is spatially localized and describes a Kelvin-Helmholtz-type instability of the shear-layer across the cavity.

In contrast to the unstable eigenspace, the stable subspace (consisting of modes with $\sigma<0)$ is high-dimensional; see table 1 . In addition, while the unstable global modes had a clear physical explanation in terms of observable instabilities, the interpretation of stable modes is far less obvious. More insight can be gained by computing the 


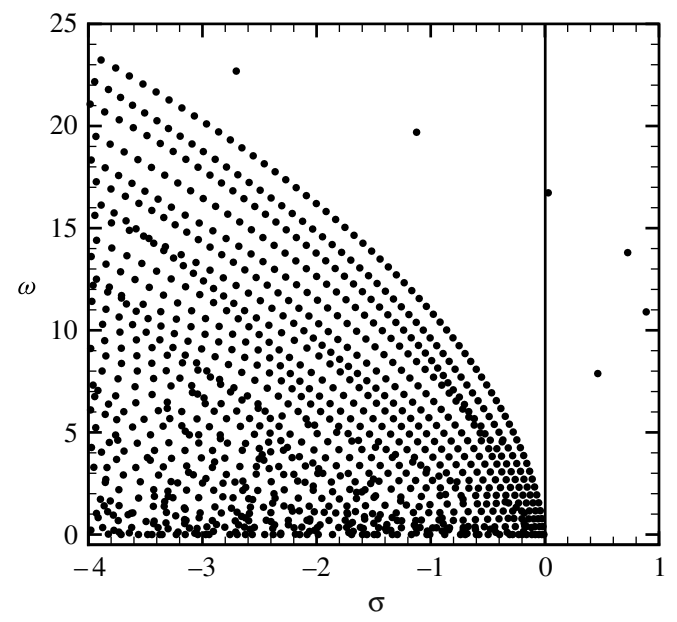

FIGURE 3. Global spectrum for flow over an open cavity at $R e=7500$.

(a)

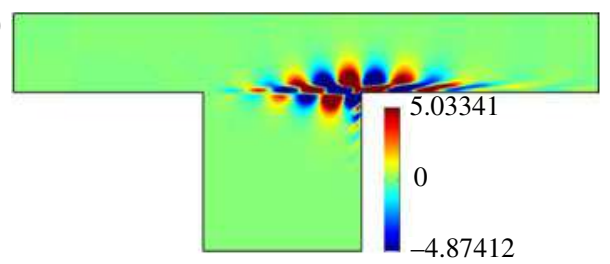

(b)

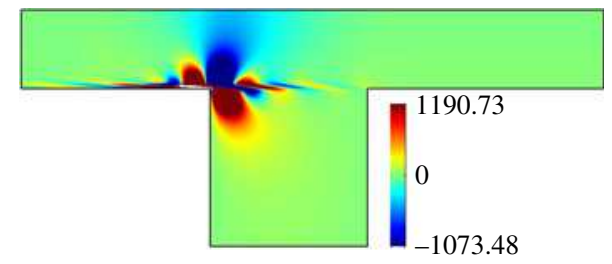

FIGURE 4. Selected direct and adjoint global modes for flow over an open cavity at $R e=7500$. (a) Most unstable direct global mode, $(b)$ adjoint global mode associated with the most unstable direct global mode in $(a)$. Both modes are visualized by contours of the streamwise velocity component.

energy-weighted $x$-centroid $x_{c}$ for each global mode defined by

$$
x_{c}=\iint_{\Omega} x e \mathrm{~d} x \mathrm{~d} y / \iint_{\Omega} e \mathrm{~d} x \mathrm{~d} y
$$

with $e$ the energy of the global mode and $\Omega$ denoting our computational domain. By definition, this quantity falls within the interval $-1.2 \leqslant x_{c} \leqslant 2.5$. The global spectrum, coloured by the centroid $x_{c}$, is shown in figure 5 and indicates the energy-weighted location of global modes within the computational domain.

Two principal groups of global modes can be distinguished: (i) modes located near the cavity with $0 \leqslant x_{c} \leqslant 1$ and (ii) modes located near the outflow boundary of the domain with $x_{c} \approx 2.5$. Eigenvalues for the former group can be found in the upper right part of the spectrum. These modes describe the stable dynamics of the motion 
(a)

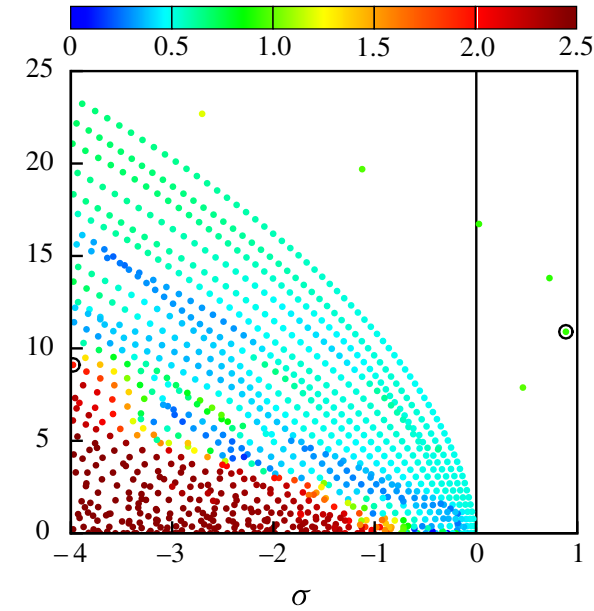

(c)

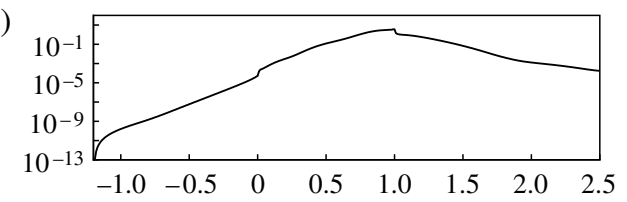

(e)

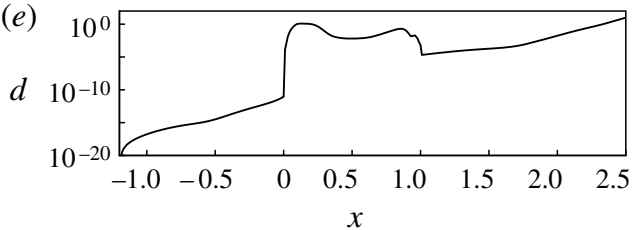

(b)

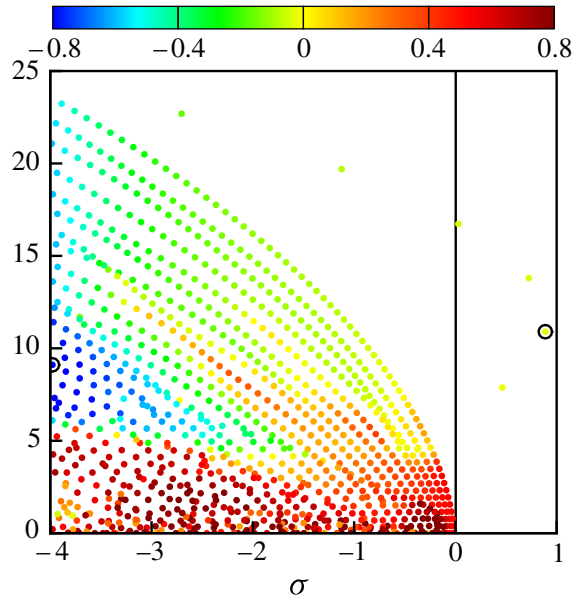

(d)

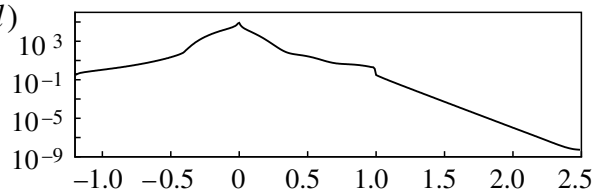

$(f)$

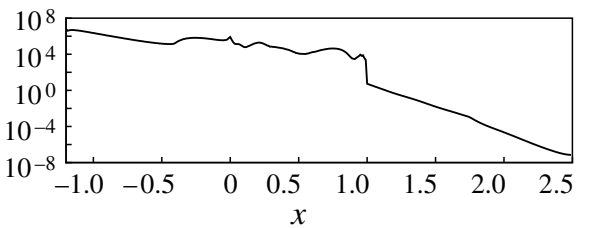

FIGURE 5. (a) Global spectrum colour-coded by the $x$-centroid of associated global modes; (b) global spectrum colour-coded by the $x$-centroid of the associated adjoint global modes. $(c, e)$ Energy density versus the streamwise position for two direct global modes, $(c)$ being the least stable direct global mode and $(e)$ a strongly damped $(\sigma \approx-4)$ direct global mode; both modes are ringed in $(a)$. $(d, f)$ Energy density versus the streamwise position for the two associated adjoint global modes, $(d)$ being the least damped adjoint global mode and $(f)$ a strongly damped $(\sigma \approx-4)$ adjoint global mode; both modes are ringed in $(b)$.

inside the cavity as well as the unstable motion of the shear layer at the top of the cavity. Eigenvalues for the latter group (with $x_{c} \approx 2.5$ ) are mainly located near the left part of the $(\sigma, \omega)$-plane at higher damping rates; their corresponding global modes are pinned to the right edge of the computational domain. Surprisingly few global modes have their energy-weighted centroid in the interval $1 \leqslant x_{c} \leqslant 2.5$, thus leaving essentially only global modes linked to either the cavity dynamics or the outflow boundary. A similar picture arises for the adjoint global modes: a first group of modes, whose centroid is located inside the cavity, can be distinguished from a second group of highly damped modes, whose centroid coincides with the inlet boundary at $x=-1.2$.

The localization of the global modes near and inside the cavity and at the outflow boundary is even better illustrated by computing the energy density $d$ of a global mode versus the streamwise coordinate. The energy density is defined as the kinetic energy of a mode at a fixed streamwise location integrated over the vertical 
coordinate, i.e. $\mathrm{d}(x)=\int e(x, y) \mathrm{d} y$. Results are given in figure $5(c, e)$. Cavity modes, with significant energy density values in the interval $0 \leqslant x \leqslant 1$, rapidly decrease in energy density away from the cavity; they show strong localization within our computational domain. Global modes located at the outlet, on the other hand, show only low to moderate energy densities near the cavity and exponential growth over many decades toward the outflow boundary. Similar observations can be made for the corresponding adjoint global modes (figure $5 d, f$ ): spatially localized adjoint cavity modes coexist with adjoint modes with strong exponential growth toward the inlet boundary.

The above analysis raises the question of the physical significance of stable global modes, in particular, in the light of the fact that increasing the computational domain will influence the stable global modes and pin them anew against the extended computational boundary. In this sense, the majority of stable global modes are linked to numerical details, in particular, to the discretization and location of outflow boundaries (see Sipp et al. 2010, for more details).

\subsection{Selection criteria for inclusion in a reduced model}

At this point, the global modes and their adjoint counterparts are available for the design of a reduced-order model and a feedback control strategy. It remains to be decided, however, which of the global modes will be included in the reduction basis $V$ (and the associated basis $W$ ). We recall that all unstable modes have to be included; for the selection of global modes from the stable half-plane a criterion has to be specified. Even though many options exist and a clear choice is far from obvious, we will concentrate on four criteria that have either appeared in previous studies (Åkervik et al. 2007; Barbagallo et al. 2009; Ehrenstein et al. 2010) or can be argued for mathematically. In the following subsections we will introduce and discuss these criteria and present their advantages, limitations and deficiencies. Arguments will be made and presented for the case of $R e=7500$; it is important to stress, however, that the results extend qualitatively to lower Reynolds numbers.

\subsubsection{Damping rate}

The first criterion is based on the damping rate of the global modes and employs the argument that modal structures with large decay rates are dynamically less relevant than modes that are only weakly damped. This criterion has been applied by Åkervik et al. (2007) in their study of flow over a shallow cavity. This criterion is particularly convenient from a numerical viewpoint as highly damped global modes are difficult to compute by iterative means - even after applying spectral transformations. An obvious flaw of this criterion is the fact that modes are selected on the basis of their dynamics rather than their controllability, observability or their contribution to the input-output behaviour between actuator and sensor. Figure 6(a) displays the stable part of the global spectrum for $R e=7500$, coloured by the growth rate. Using a criterion based on the damping rate, global modes of darker colours are included first in the basis $V$; global modes of lighter colours are included last.

\subsubsection{Contribution to the input-output behaviour}

Acknowledging the importance of controllability and observability of each individual global mode, the criterion (see e.g. Antoulas 2005; Bagheri et al. 2009b; 


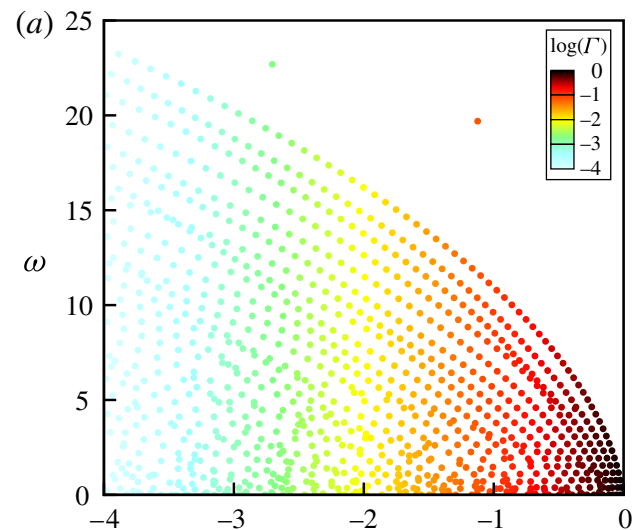

(b) 25

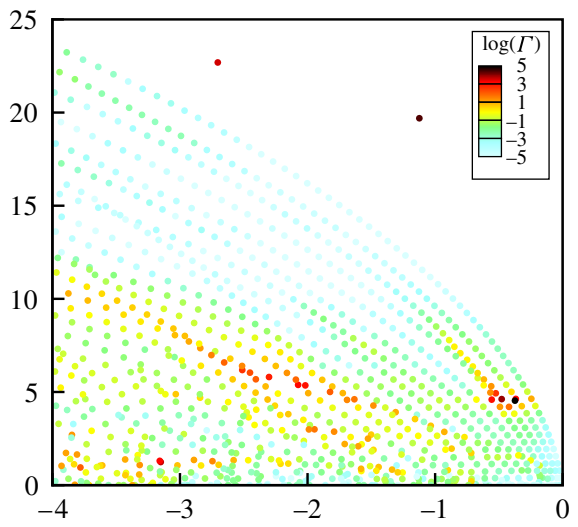

(c)

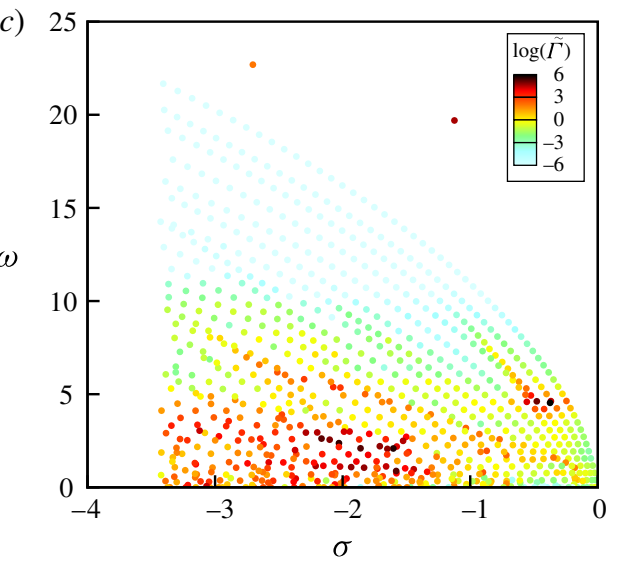

(d)

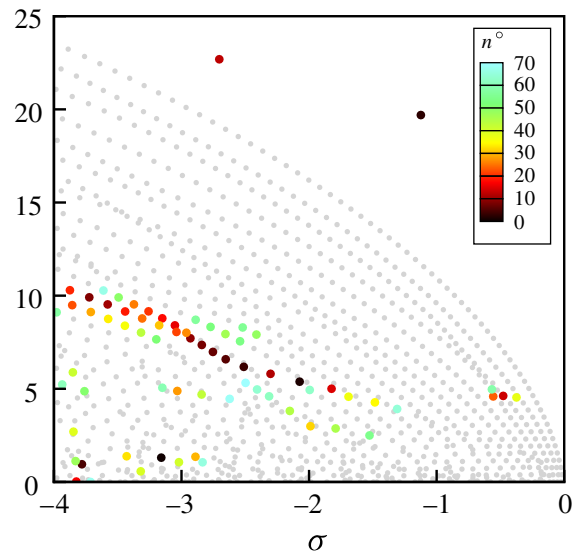

FIGURE 6. Identification of global modes by colour-coding according to the four respective criteria (see text): (a) growth rate, $(b)$ contribution to input-output behaviour, $(c)$ orthogonal projection, and $(d)$ 'quasi-optimal' stability ranking. Only the stable part of the spectrum is displayed; the Reynolds number is $R e=7500$.

Barbagallo et al. 2009)

$$
\Gamma_{i}=\frac{\left|\hat{B}_{i}\right|\left|\hat{C}_{i}\right|}{\left|\lambda_{i}\right|}
$$

has been introduced, where $\hat{B}_{i}$ is the projection of the actuator onto the $i$ th global mode, $\hat{C}_{i}$ is the measured component of the $i$ th global mode and $\lambda_{i}$ is the decay rate of the $i$ th global mode. This criterion identifies modes that are simultaneously responsive to control efforts, can be measured by the sensor and are only weakly damped.

Figure $6(b)$ depicts the global spectrum, again for $R e=7500$, coloured by the criterion $\Gamma$. Darker colours indicate modes with high values of $\Gamma_{i}$; these modes would be preferentially included in a reduced-order model based on the $\Gamma$-selection criterion. Modes with lighter colours have lower associated values of $\Gamma_{i}$ and would tend to be neglected in a reduction basis. The first modes selected according to the criterion are clustered near $\lambda=(\sigma, \omega) \approx(-0.4,4.5),(-1.1,19.7)$ and $(-2.7,22.7)$. Close analysis (see also Barbagallo et al. 2009) reveals that the corresponding modes show a spatial structure similar to the unstable global modes, that is, linking actuator 
and sensor by a chain of vortical elements. More problematic is the fact that a substantial number of modes with high damping rates appear to contribute to the input-output behaviour; even modes near our cut-off damping rate of $\sigma=-4$ seem to be important, which suggests that even higher damping rates would have been favoured, had they been available.

\subsubsection{Orthogonal projection}

The third criterion makes use of an additional projection and has been suggested in Ehrenstein et al. (2010). It has been shown to improve results obtained by only applying the bi-orthogonal projection introduced earlier. The method combines the bi-orthogonal projection for the unstable subspace with a least-squares projection for the stable supplementary one. The central idea stems from the observation by Ehrenstein et al. (2010) that the actuator expressed in the bi-orthogonal projection is spatially rather different from its original form. For this reason, they suggest a least-squares projection method for the actuator as it minimizes the error between the original and projected actuator. Following this concept, the global modes are ranked using the criterion

$$
\tilde{\Gamma}_{i}=\frac{\left|\tilde{B}_{i}\right|\left|\hat{C}_{i}\right|}{\left|\lambda_{i}\right|}
$$

where the coefficient $\tilde{B}_{i}$ is now based on the least-squares projection. The coefficients are used in (4.7) as well as in the reduced-order model (3.3); hence the Kalman gains and the estimator equation are based on $\tilde{B}_{i}$. Note that, as in Ehrenstein et al. (2010), the coefficients $\tilde{B}_{i, i=1 \ldots n}$ are first computed to rank the set of $n=1601$ least stable global modes; then, for each model consisting of $p \leqslant n$ global modes, the coefficents $\widetilde{B}_{i, i=1 \ldots p}$ are computed again and used in (3.3).

Figure 6(c) shows the global spectrum $(R e=7500)$ coloured by the above criterion $\tilde{\Gamma}$ to indicate a classification of global modes (from darker to lighter colours) for their inclusion in the reduced-order model. A similar general tendency is observed as in figure 6(b): preference of modes that resemble the globally unstable modes and of highly damped, low-frequency modes. In addition, the least-squares projection becomes computationally more sensitive for modes at higher damping rates which allowed us to accurately evaluate the criterion $\tilde{\Gamma}$ only for modes with $\sigma>-3.4$. This marked numerical sensitivity has been observed for all Reynolds numbers considered in this study. It is also worth pointing out that due to the two independent projections (bi-orthogonal and least-squares), the resulting reduced-order model cannot be written as a single projection of the initial system onto a set of global modes; rather, the coefficients $\widetilde{B}_{i}$ enter the dynamics given by (3.3).

\subsection{4. 'Quasi-optimal' stability ranking}

The fourth criterion is included as a demonstration tool for the ability of reduced models based on global modes to yield a stable compensated system. As will become clearer in the following sections, the fully compensated system, i.e. the plant coupled with a reduced estimator and controller, suffers from instabilities when global modes are used in the reduced-order model. These instabilities render the ROM ineffective and illustrate that global modes are inadequate for our closed-loop control application. The fourth criterion aims to explore the best possible choice of global modes such that stability of the fully compensated system is approached as swiftly as possible. As such the criterion provides a benchmark for what is possible with a judicious choice of global modes. It is computed iteratively and a posteriori as follows. We start with a 
reduced-order model comprising only the unstable modes and compute the growth rate $\sigma$ of the compensated system (plant plus reduced-order estimator plus reduced-order controller). Next we search through all available stable global modes and add the one to the reduction basis $V$ which results in the largest reduction of $\sigma$ for the augmented compensated system. This procedure is repeated, thus building a sequence of global modes where each added global mode improves the stability of the compensated system more than any of the remaining modes. Non-normal effects from an interaction of the included modes are not considered in this criterion, even though the individual modes account for non-normal effects in the global mode expansion. Since we are interested in the long-term stability of the compensated system, the concentration on eigenvalues only can be justified.

We note that for a model with $p$ stable modes, the truly optimal ranking according to stability would be given by the best combination of $p$ modes chosen from the complete set of $n$ stable modes. The number of admissible combinations is $n ! /(p !(n-p) !)$ which far exceeds our computational means. For this reason, the 'quasi-optimal' sequential algorithm for building a reduction basis has been chosen.

Figure $6(d)$ illustrates the global spectrum coloured by the ordering based on the above criterion. The eigenvalues selected by the 'quasi-optimal' stability procedure are reminiscent of some of the eigenvalues chosen by the $\Gamma$-criterion (see figure $6 b$ ). On the other hand, many global modes with high values of $\Gamma$ are not selected by the stability criterion, a feature that will be discussed in more detail later.

\subsection{Final form of the compensator using a reduced-order model based on global modes}

We conclude this section by stating the equations governing the compensator. In particular, we emphasize the partitioning of the reduced-order model into an unstable (indicated by subscript $u$ ) and stable (indicated subscript $s$ ) part. The control gain $\hat{K}$ as well as the Kalman gain $\hat{L}$ have been computed in the small gain limit (see e.g. Burl 1999) which introduces zero matrices since the estimator and controller only act on the unstable subspace. For more details and an explicit formulation of the Riccati equations in this limit, the reader is referred to Barbagallo et al. (2009). We have

$$
\begin{aligned}
\frac{\mathrm{d}}{\mathrm{d} t}\left(\begin{array}{c}
\hat{\boldsymbol{Y}}_{u} \\
\hat{\boldsymbol{Y}}_{s}
\end{array}\right)= & \left(\begin{array}{cc}
\hat{\boldsymbol{\Lambda}}_{u} & 0 \\
0 & \hat{\boldsymbol{\Lambda}}_{s}
\end{array}\right)\left(\begin{array}{c}
\hat{\boldsymbol{Y}}_{u} \\
\hat{\boldsymbol{Y}}_{s}
\end{array}\right)+\left(\begin{array}{c}
\hat{\boldsymbol{B}}_{2 u} \\
\hat{\boldsymbol{B}}_{2 s}
\end{array}\right)\left(\begin{array}{ll}
\hat{K}_{u} & 0
\end{array}\right)\left(\begin{array}{c}
\hat{\boldsymbol{Y}}_{u} \\
\hat{\boldsymbol{Y}}_{s}
\end{array}\right) \\
& \left.\left.-\left(\begin{array}{c}
\hat{\boldsymbol{L}}_{u} \\
0
\end{array}\right)\left[\begin{array}{ll}
\hat{\boldsymbol{Y}}_{u} \\
\hat{\boldsymbol{Y}}_{s}
\end{array}\right)\right],\left(\begin{array}{ll}
\hat{\boldsymbol{C}}_{u} & \hat{\boldsymbol{C}}_{s}
\end{array}\right)\right] \\
u & =\left(\begin{array}{ll}
\hat{K}_{u} & 0
\end{array}\right)\left(\begin{array}{c}
\hat{\boldsymbol{Y}}_{u} \\
\hat{\boldsymbol{Y}}_{s}
\end{array}\right) .
\end{aligned}
$$

The complete compensator is now set up. In the following section we will present open-loop tests based on the approximation of the full transfer behaviour by the reduced model and closed-loop tests based on the stability properties of the compensated system. The four selection criteria will be investigated for the representative case of cavity flow at $R e=7500$.

\section{Performance evaluation of reduced-order models for $R e=7500$}

Two performance measures will be used to assess the performance of the reducedorder models based on global modes. The first measure is concerned with the accurate 
representation of the input-output behaviour of the full system by the reduced model. The approximation error is quantified by the mismatch of the true transfer function and the transfer function of the reduced system, measured in the $\mathscr{H}_{2}$ - or $\mathscr{H}_{\infty}$-norm. The second measure probes the ability of the reduced model to yield a stable compensated system. In this case, the eigenvalue of the coupled plant-compensator system with the largest growth rate will be used as a performance indicator.

\subsection{Open-loop performance evaluation}

It is generally acknowledged that a successful control design requires the representation of the correct input-output behaviour by the reduced model. Since there is no choice in selecting unstable global modes, only the accuracy of modelling the stable subspace dynamics needs to be addressed. The governing equations for the stable subspace dynamics in state-space form can be written as

$$
\begin{gathered}
Q \frac{\mathrm{d} \boldsymbol{X}}{\mathrm{d} t}=A_{s} \boldsymbol{X}+Q \boldsymbol{B}_{s} u, \\
m=\boldsymbol{C} \boldsymbol{X},
\end{gathered}
$$

where $\boldsymbol{B}_{s}$ denotes the actuator projected onto the stable subspace. The input-output dynamics is computed numerically by imposing a Dirac impulse $u(t)=\delta(t)$ as the control input and subsequently measuring the signal $m(t)$ at the sensor location. Since, by definition, the subspace system given by $A_{s}$ is stable, the signal $m(t)$ will eventually decay as $t \rightarrow \infty$. Transforming this impulse response into the frequency domain according to $\hat{m}(\omega)=\int_{0}^{\infty} m(t) \exp (-\mathrm{i} \omega t) \mathrm{d} t$ (for a causal system), we obtain the transfer function $H(\omega)=\hat{m}(\omega)$ which quantifies the response of the system to a harmonic forcing at a frequency $\omega$. In figure 7 the transfer functions (in the frequency domain) are shown. The transfer function curves correspond to the amplitude part of a Bode diagram and exhibit two (nearly constant) plateaus for $\omega \in[0,3] \cup[20,25]$ and $\omega \in[5,19]$. The latter range approximately corresponds to the frequency range of the unstable modes. We also notice an abrupt drop around $\omega \approx 4$, causing a nearly vanishing response at this frequency and suggesting the presence of nearly unobservable states.

Once the transfer function of the full system has been computed from a Fourier transform of the impulse response, we can juxtapose the transfer functions of the reduced-order models and assess the quality of the approximation as more and more global modes are added to the reduction basis according to one of the four criteria.

In figure 7 the amplitude of the transfer function is displayed for reduced-order models using the selection criteria described in $\S 4.3$. The transfer functions have been computed using the expression $\hat{H}(\omega)=\hat{\boldsymbol{C}}\left(\mathrm{i} \omega l-\hat{\boldsymbol{A}}_{s}\right)^{-1} \hat{\boldsymbol{B}}_{s}$. The different reduced-order models are identified by colours (see the figure legends) and correspond to a specific number of included global modes ranked by the respective criterion. The transfer function of the original (full) system is given in black. At a first glance it can be observed that - perhaps with the exception of the 'quasi-optimal' stability criterion - none of the four selection criteria produces satisfactory results even if more than a thousand global modes are included.

To quantify the degree of approximation of the original transfer function we consider the $\mathscr{H}_{2}$ - and $\mathscr{H}_{\infty}$-norms defined as

$$
\|H\|_{2}=\left(\int_{0}^{25}|H(\omega)|^{2} \mathrm{~d} \omega\right)^{1 / 2},
$$




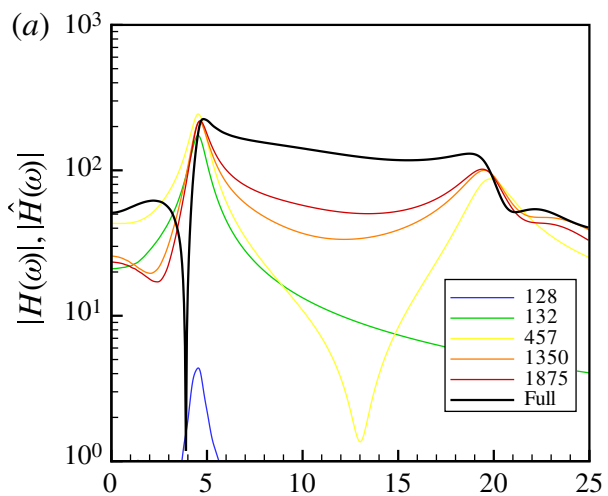

(b)
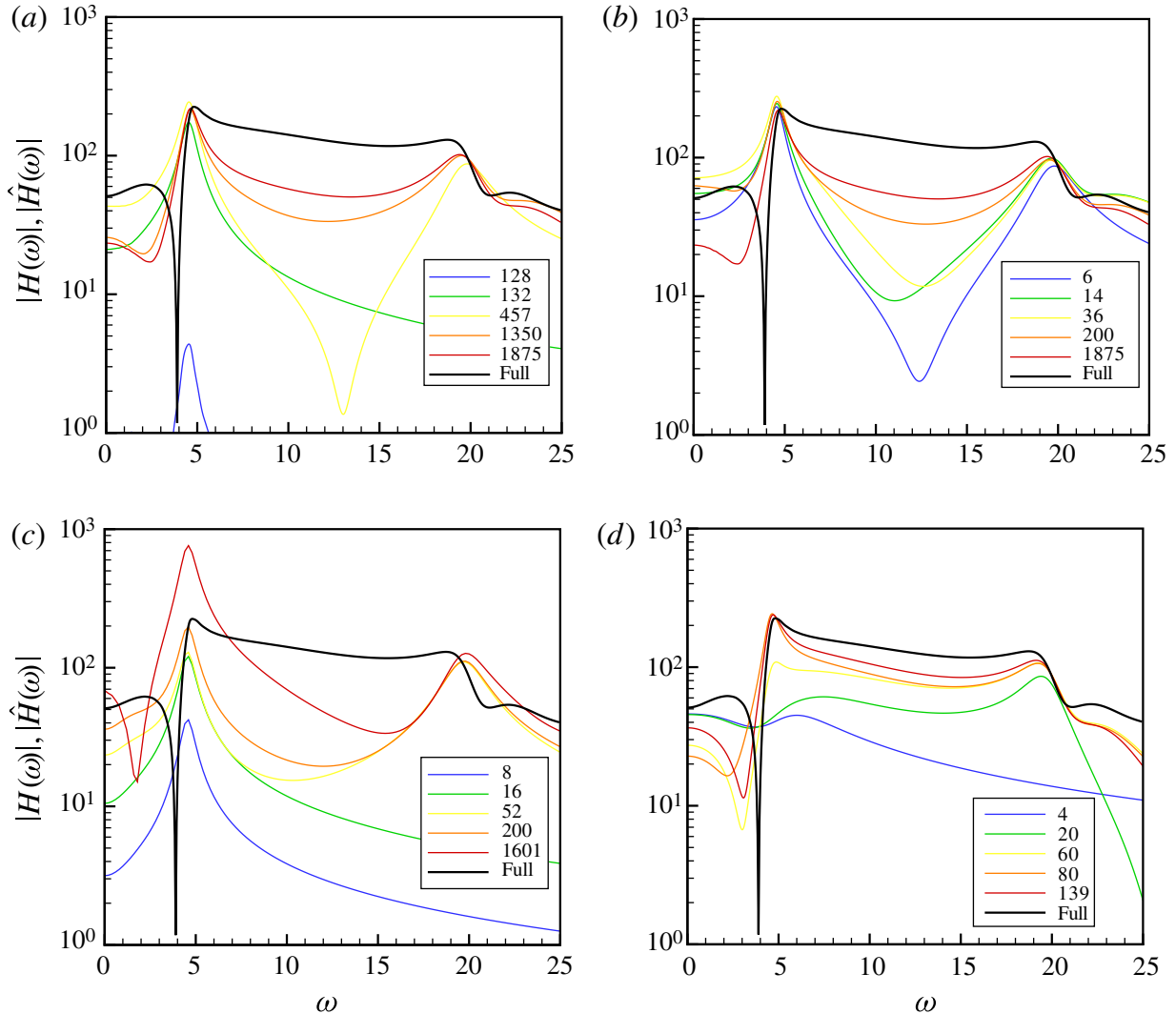

(d)

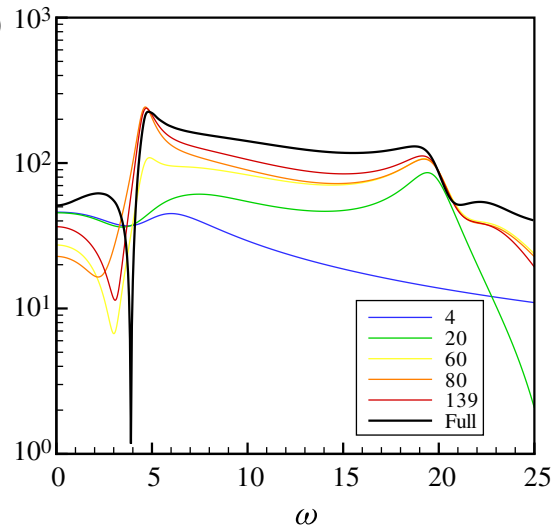

FIGURE 7. Approximation of the modulus of the exact transfer function (in black) as global modes are added to the reduction basis $V$ according to the four selection criteria; $(a)$ adding modes based on damping rate, $(b)$ adding modes according to their contribution to the input-output behaviour, $(c)$ adding modes using orthogonal projections, $(d)$ adding modes based on the 'quasi-optimal' stability ranking. The Reynolds number is $R e=7500$.

$$
\|H\|_{\infty}=\sup _{\omega}|H(\omega)| .
$$

The $\mathscr{H}_{2}$-norm measures the overall error over a given frequency range, whereas the $\mathscr{H}_{\infty}$-norm concentrates on the worst error that occurs in a frequency range. These measures account for the degree of approximation between the full and reduced-order transfer functions. In figure 8 the $\mathscr{H}_{2}-$ and $\mathscr{H}_{\infty}$-errors are displayed and corroborate the findings from figure 7. In particular, the ranking based on orthogonal projections of the controller shows rather discouraging results.

Figure 7(a) displays the amplitude of the transfer function for reduced-order models of dimension 128, 132, 457, 1350 and 1875 where the modes have been ranked by damping rate (first criterion in $\S 4.3$ ). As the dimension of the reduced models is increased, the transfer functions of the reduced models approach the transfer function of the original system. This effect is also visible in figure 8 (open squares); in both norms, the error is gradually decreasing. The convergence, however, is rather erratic: for example, the transfer functions of a reduced model of order 128 and of order 132 are entirely different, even though they differ merely by four stable global modes that have been added to the latter model. 

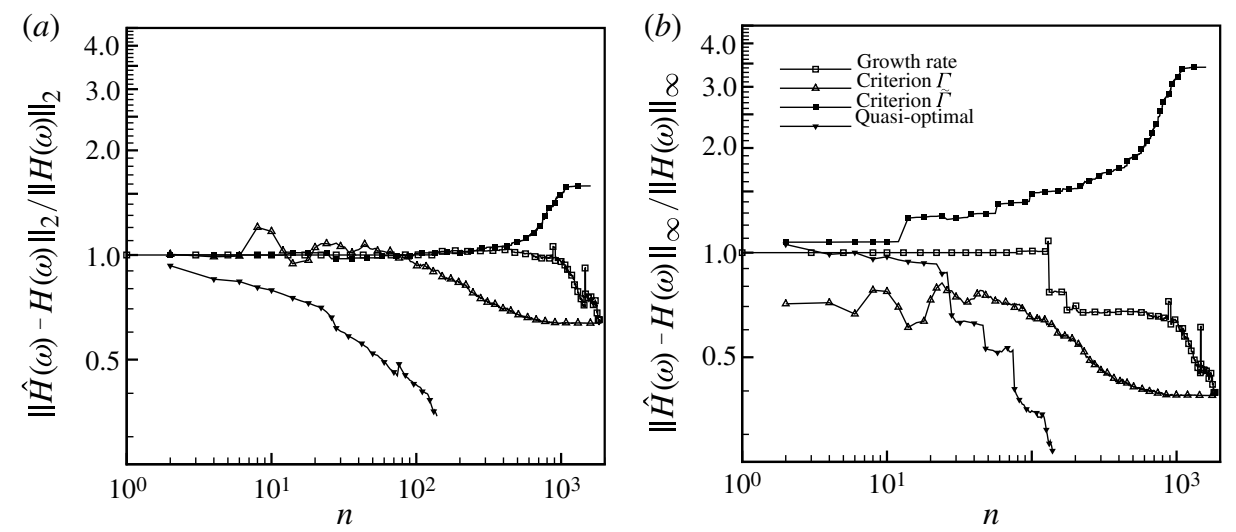

FIGURE 8. Relative approximation error of the full transfer function as global modes are added to the reduction basis $V$ according to the four criteria (see text). (a) Relative error measure in the $\mathscr{H}_{2}$-norm, $(b)$ relative error measured in the $\mathscr{H}_{\infty}$-norm. The Reynolds number is $R e=7500$.

In figure $7(b)$ we see the amplitude of the transfer function for reduced-order models of dimension 6,14, 36, 200 and 1875; this time, the modes have been ranked by the input-output criterion $\Gamma$ (see $\S 4.3$ ). Similarly to the previous case, the transfer functions approach the one of the original system, but better approximations are achieved for smaller numbers of included modes. This validates the relevance of the $\Gamma$-criterion in selecting global modes for the reduction basis $V$. The first few modes are assumed to contribute significantly to the input-output behaviour; it may thus seem confusing that the relative errors (upward-pointing triangles in figure 8) do not decrease as the first modes are added. This phenomenon is due to the fact that the difference between the full transfer function and the transfer function based on $p$ global modes is represented by a poorly converging series of the form

$$
H-\hat{H}=\sum_{j=p+1}^{N} \frac{\hat{C}_{j} \hat{B}_{j}}{\mathrm{i} \omega-\lambda_{j}},
$$

where each term in the sum is of considerable size due to the non-normality of our system. Mutual cancellation by terms is observed, and bounds based on the magnitude of these terms quickly become unsharp. These effects and their link to the non-normality of the system are further explained in Barbagallo et al. (2009).

Figure $7(c)$ depicts the amplitude of transfer functions for reduced models of dimension 8, 16, 52, 200 and 1601 where the modes have been ranked by the criterion $\tilde{\Gamma}_{i}$ (see $\S 4.3$ ). Even though the transfer functions of figures $7(a)$ and $7(b)$ show poor convergence towards the exact system as more unstable global modes are added, the $\tilde{\Gamma}$-criterion yields approximate transfer functions that lack convergence altogether (see filled squares in figure 8).

Finally, figure $7(d)$ shows the amplitude of the transfer function for reduced models of dimension 4, 20,60,80 and 139 where the modes have been ranked according to the 'quasi-optimal' stability criterion (see $\S 4.3$ ). As the dimension of the reducedorder model increases, we observe an adequate convergence of the reduced transfer functions toward the original one (in particular, for the frequency interval $\omega \in[5,20]$ ). This behaviour may indicate that the transfer function does not have to approximate 
the original transfer function over the entire frequency range - an issue that will be further investigated in $\S 5.3$.

Overall, we confirm that the relative error norms based on the fourth criterion are markedly lower than the error norms for the previous three criteria. This indicates that a particular ordering of global modes may indeed produce an effective reduced-order model; an a priori and definitive selection criterion, however, may be difficult to devise. The study of the open-loop behaviour of reduced-order models, expressed by their transfer function, has established that the criterion for selecting global modes that are to be included in a reduced-order model is pivotal. Intuitive concepts, such as growth rate, are often misleading and produce ineffective models; even more sophisticated criteria cannot guarantee robust success over a broad range of flow parameters. The underlying reason for this may lie in the fact that most of the stable global modes carry little physical meaning.

In the following section, the more categorical test of a reduced-order model, namely the stabilization of the full compensated system, will be presented.

\subsection{Performance of the closed-loop system}

For supercritical Reynolds numbers, the objective of the LQG-control loop, shown in figures 1 and 2, is to suppress the instabilities in the system. To test whether the closed-loop system has succeeded in this, the growth rate $\sigma_{\max }$ of the least stable eigenvalue of the compensated system is the natural quantity to evaluate. If at least one unstable eigenvalue exists, the controlled system is still unstable. If all eigenvalues of the compensated system are stable, the originally unstable system has been stabilized (in the asymptotic limit $t \rightarrow \infty$ ).

The compensated system is constructed by coupling the plant (2.7) to the compensator (4.8). The spectrum of this coupled system then provides information about the success of our closed-loop control design as well as our model reduction efforts. Since the plant contains a very large number of degrees of freedom (in our case, nearly one million), spectral information about the coupled system is challenging to extract. Instead, we will follow Barbagallo et al. (2009) and reduce the computational costs by replacing the full plant model (2.7) by a reduced-order model based on unstable global modes and proper orthogonal decomposition (POD) modes for the stable subspace; see the Appendix for further details. A sufficient number of POD modes has to be taken into account to accurately represent the plant dynamics and its response behaviour. With this substitution, the compensated system can then be written as

$$
\frac{\mathrm{d}}{\mathrm{d} t}\left(\begin{array}{c}
\hat{\boldsymbol{X}}_{u}^{g m} \\
\hat{\boldsymbol{X}}_{s}^{p o d} \\
\frac{\hat{\boldsymbol{Y}}_{u}^{g m}}{\hat{\boldsymbol{Y}}_{s}^{g m}}
\end{array}\right)=\hat{\boldsymbol{A}}_{c}\left(\begin{array}{c}
\hat{\boldsymbol{X}}_{u}^{g m} \\
\hat{\boldsymbol{X}}_{s}^{\text {pod }} \\
\hat{\boldsymbol{Y}}_{u}^{g m} \\
\hat{\boldsymbol{Y}}_{s}^{g m}
\end{array}\right)
$$

with

$$
\hat{\boldsymbol{A}}_{c}=\left(\begin{array}{cccc}
\hat{\boldsymbol{\Lambda}}_{u}^{g m} & 0 & \hat{\boldsymbol{B}}_{u}^{g m} \hat{\boldsymbol{K}}_{u}^{g m} & 0 \\
0 & \hat{A}_{s}^{\text {pod }} & \hat{\boldsymbol{B}}_{s}^{\text {pod }} \hat{\boldsymbol{K}}_{u}^{g m} & 0 \\
-\hat{L}_{u}^{g m} \hat{\boldsymbol{C}}_{u}^{g m} & -\hat{L}_{u}^{g m} \hat{\boldsymbol{C}}_{s}^{\text {pod }} & \hat{\boldsymbol{\Lambda}}_{u}^{g m}+\hat{\boldsymbol{B}}_{u}^{g m} \hat{\boldsymbol{K}}_{u}^{g m}+\hat{\boldsymbol{L}}_{u}^{g m} \hat{\boldsymbol{C}}_{u}^{g m} & \hat{\boldsymbol{L}}_{u}^{g m} \hat{\boldsymbol{C}}_{s}^{g m} \\
0 & 0 & \hat{\boldsymbol{B}}_{s}^{g m} \hat{K}_{u}^{g m} & \hat{\boldsymbol{\Lambda}}_{s}^{g m}
\end{array}\right)
$$




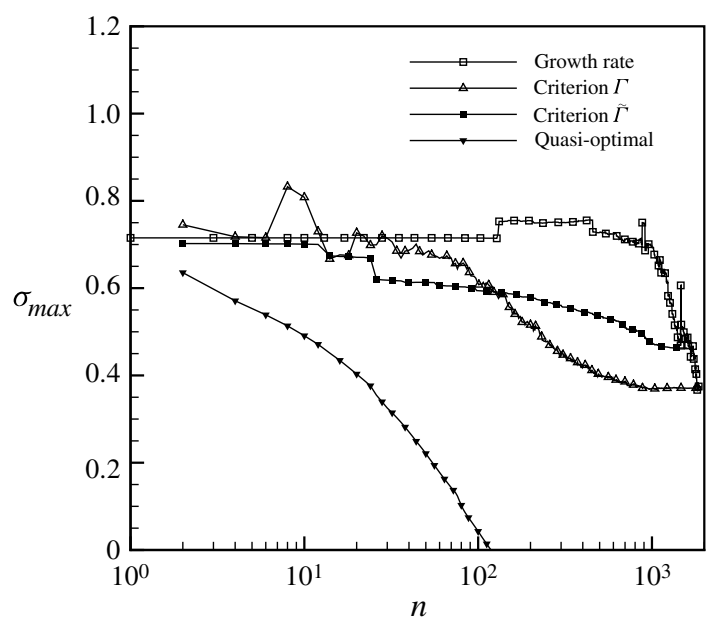

FIGURE 9. Growth rate of least stable eigenvalue of the compensated system as global modes are added to the reduction basis $V$ according to the four criteria (see text). The Reynolds number is $R e=7500$.

Superscripts $g m$ and pod denote whether the respective matrix has been reduced by global or POD modes; the subscripts $u$ and $s$ stand, as before, for the unstable or stable subspace, respectively. The above system then describes the compensated dynamics, and the growth rate of the least stable eigenvalue of the matrix $\hat{A}_{c}$ indicates failure or success of the reduced-order LQG controller to stabilize the otherwise unstable system. We will now use this technique to evaluate the closed-loop performance of reduced-order models based on selected global modes, again for a representative Reynolds number of $R e=7500$.

The eigenvalues of the compensated system matrix $\hat{A}_{c}$ have been determined. Direct methods could be employed since the original system matrix $A$ for the plant has been replaced by a lower-dimensional matrix based on POD modes. The growth rate $\sigma_{\max }$ of the least stable eigenvalue determines the stability property of the compensated system. Figure 9 displays this growth rate $\sigma_{\max }$ as a function of the number $n$ of stable global modes included in the reduced-order model. The selection and ranking criteria introduced earlier have been used, and the various stability characteristics for each criterion are shown in the figure.

The compensated system can only be stabilized by the 'quasi-optimal' ranking procedure (downward-pointing triangles). In this case, stabilization of the flow is achieved, once more than 120 stable global modes are used in the reduced-order model. We observe a monotonic decrease of $\sigma_{\max }$ which is expected since at each $n$ only the global mode that maximally decreases $\sigma_{\max }$ is added to the reduced-order model.

Models using the growth rate criterion (open squares) also show a decrease in $\sigma_{\max }$ as more stable modes are taken into account. Within the limits of our available modes, however, a stable compensated system could not be attained. An interesting observation is that the growth rate curve (open squares) in figure 9 is highly correlated with the equivalent curve representing the relative $\mathscr{H}_{2}$-error (open squares in figure $8 a$ ), which appears to suggest a link between a better approximation (in the $\mathscr{H}_{2}$-sense) of the open-loop input-output behaviour and an improved stability of the closed-loop system. 
Similar results are obtained when using a ranking of the stable global modes according to the $\Gamma$-criterion (upward-pointing triangles). However, as in the open-loop test, an improvement of the stability properties of the compensated system emerges substantially earlier compared to the ranking based on the growth rate. Nevertheless, within the limit of available global modes, no stabilization could be achieved.

Lastly, the results corresponding to the $\tilde{\Gamma}$-criterion (using an orthogonal projection for the actuator) are displayed (filled squares). The behaviour of $\sigma_{\max }$ displays a tendency similar to the previous case based on the $\Gamma$-criterion: for $p \leqslant 100$ it shows performance superior to the $\Gamma$-criterion; for larger values of $p$, however, a more unstable compensated system results. The favourable properties of this projection reported in Ehrenstein et al. (2010) could not be recovered for our flow configuration.

\subsection{A frequency-restricted norm measuring open-loop behaviour}

The similarities between the curves of $\sigma_{\max }$ (figure 9), which express the performance of the compensator, and the curves of the relative $\mathscr{H}_{2}$-error (figure 8 ), which express the accuracy of capturing the input-output behaviour of the original system, are striking and warrant further investigation.

Special attention has to be paid to the choice of norm when evaluating the open-loop characteristics. The $\mathscr{H}_{\infty}$-norm is commonly adopted in control and model reduction applications since rigorous error bounds are available for this norm (see e.g. Antoulas 2005). Our study, however, indicates that the performance of the closed-loop system (figure 9) is much more closely linked to the $\mathscr{H}_{2}$-errors (figure $8 a$ ) than the $\mathscr{H}_{\infty}$-errors (figure $8 b$ ) of the reduced transfer function. This suggests that the overall behaviour of the reduced-order model may be more relevant than its worse departure from the exact behaviour at a particular frequency.

Another remarkable observation is that, according to the $\mathscr{H}_{2}$-error of the open-loop behaviour, the reduced-order models based on the $\tilde{\Gamma}$-criterion are expected to perform poorly: the error curve (filled squares) is close to and, eventually, above the curve representing models based on the growth-rate criterion (open squares) in figure $8(a)$. Nevertheless, $\tilde{\Gamma}$-models stabilize the system noticeably better than models using the growth-rate criterion, as can be seen in figure 9. This apparent inconsistency may give insight into which frequency range of the open-loop transfer function has to be captured sufficiently by the reduced-order model to yield improved stability properties for the closed-loop system. None of the reduced-order models reproduces the full transfer function behaviour adequately at low frequencies (see figure 7). Nevertheless, it can be observed that the instability of the compensated system is noticeably reduced as soon as the transfer function is well-approximated over a frequency range of $\omega \in[10,20]$.

This observation is in agreement with the physical understanding of the control dynamics. In an effort to eliminate an unstable mode, the controller generates an opposite structure that destructively interferes with the instability. Since the unstable modes are characterized by well-defined frequencies, the actuator also has to operate at the same frequencies. Doing so, it mainly triggers the stable part of the flow at these frequencies. As a result, the control-oriented reduced-order model must largely capture the full input-output behaviour at these frequencies. Frequencies outside this range play a subordinate role in the control law, and the transfer behaviour at these frequencies may not have to be captured as accurately. Based on this argument, we propose to evaluate the reduced-order models using a frequency-restricted $\mathscr{H}_{2}$-norm; the frequency range is chosen to include the frequencies of the unstable modes. We 


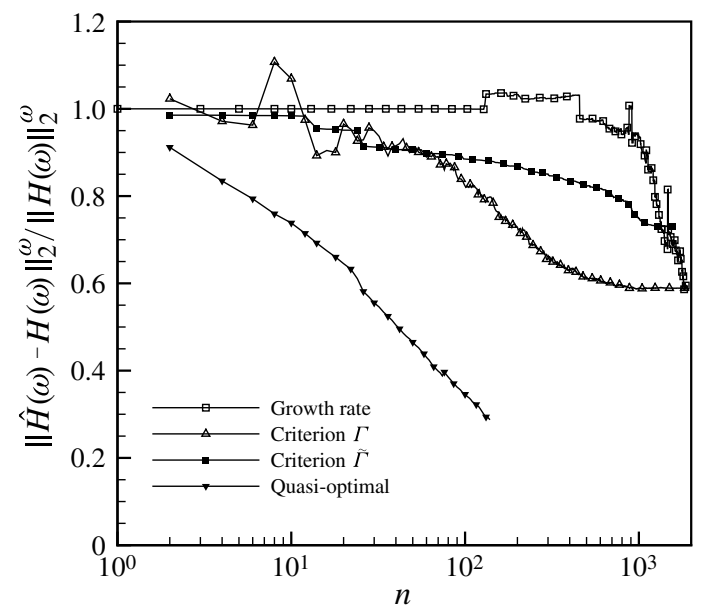

FIGURE 10. Relative approximation error of the reduced-model transfer function as global modes are added to the reduction basis $V$ according to the four criteria (see text). The relative error is measure in the frequency-restriced norm $\mathscr{H}_{2}^{\omega}$. The Reynolds number is $R e=7500$.

introduce

$$
\|H\|_{2}^{\omega}=\left(\int_{10}^{17}|H(\omega)|^{2} \mathrm{~d} \omega\right)^{1 / 2}
$$

and refer to this norm as the $\mathscr{H}_{2}^{\omega}$-norm. Alternatively, this norm can also be defined by introducing a bandpass filter as a weight function under the integral. The $\mathscr{H}_{2}^{\omega}$-error of the reduced transfer functions is plotted in figure 10. First inspection shows that all curves reflect the corresponding performance of the closed-loop system (figure 9). More importantly, the relative positions of the curves are very similar to the results in figure 9 which confirms our supposition that it is of critical importance to capture the behaviour of the original system at the frequencies where the control acts.

\subsection{A frequency-restricted reduced-order model of the stable subspace}

In previous studies of closed-loop control of flow instabilities using a reduced-order compensator (Bagheri et al. 2009a; Barbagallo et al. 2009), successful stabilization has been achieved using a reduced-order model for the entire frequency range of the stable part of the flow. Our findings from the last section, however, indicate that only the limited frequency range triggered by the actuator should be necessary to capture the input-output behaviour of the stable subspace. Under this assumption, a further dimensionality reduction of the reduced-order model is conceivable. This conjecture will be investigated by constructing a frequency-restricted POD model; the same analysis can be applied to balanced POD models.

\subsubsection{Construction of a frequency-restricted reduced-order model}

POD modes for control applications are typically computed from the eigenvectors of the controllability matrix $G_{c}$ expressed in the frequency domain (see Willcox \& Peraire 2002). We have

$$
G_{c}=\frac{1}{2 \pi} \int_{-\infty}^{\infty}(j \omega l-A)^{-1} \boldsymbol{B B}^{*}\left(-j \omega l-A^{*}\right)^{-1} \mathrm{~d} \omega .
$$


A restriction to a specified frequency range is straightforwardly implemented by modifying the integration limits of the above integral accordingly. We thus obtain, for a given frequency range $\left[-\omega_{2},-\omega_{1}\right] \cup\left[\omega_{1}, \omega_{2}\right]$, the expression

$$
\begin{aligned}
G_{c}^{\omega}= & \frac{1}{2 \pi} \int_{-\omega_{2}}^{-\omega_{1}}(j \omega l-A)^{-1} \boldsymbol{B B}^{*}\left(-j \omega I-A^{*}\right)^{-1} \mathrm{~d} \omega \\
& +\frac{1}{2 \pi} \int_{\omega_{1}}^{\omega_{2}}(j \omega I-A)^{-1} \boldsymbol{B B}^{*}\left(-j \omega I-A^{*}\right)^{-1} \mathrm{~d} \omega .
\end{aligned}
$$

Instead of explicitly constructing the matrix $G_{c}$, a snapshot method may be used to reduce the order of the eigenvalue problem for the POD-modes from the number of degrees of freedom to the number of snapshots (see Dergham et al. 2011). Alternatively, it is also possible to evaluate the Gramian (5.7) in the time domain (using temporal snapshots) by evaluating

$$
G_{c}^{\omega}=\int_{0}^{t} \boldsymbol{X}(t) \boldsymbol{X}^{*}(t) \mathrm{d} t \quad \text { with } \boldsymbol{X}(t)=\int_{0}^{t} \mathrm{e}^{A(t-\tau)} \boldsymbol{B} \frac{\sin (\pi \tau)}{\left(\omega_{2}-\omega_{1}\right) \tau} \mathrm{d} \tau .
$$

The additional term in the expression for $\boldsymbol{X}(t)$ corresponds to the transfer function of our top-hat bandpass filter. Finally, it is worth mentioning that due to the frequency truncation the reduced-order model obtained by projection onto the 'frequencyrestricted' POD modes is not guaranteed to be stable.

\subsection{Performance of the frequency-restricted reduced-order models}

Four different cases have been computed to evaluate the effect of restricting the frequency range on the performance of the reduced-order model. As before, both the deviation from the full transfer function (an open-loop measure) and the stability of the compensated system (a closed-loop measure) will be assessed. The four cases cover an increasingly restricted frequency range, starting with the reference case (case 1$)$ that takes into account all frequencies $\left(\omega_{1}=0, \omega_{2} \rightarrow \infty\right)$ and progressing to $\omega \in[4,20]$ (case 2) which considers a frequency interval larger than the frequency band of unstable modes (see figure 3), to $\omega \in[7,17]$ (case 3) which covers the frequencies of the unstable modes, to finally $\omega \in[11,14]$ (case 4) which concentrates on the frequencies of the two most unstable modes.

As a performance measure for the open-loop test we take the effectiveness of the frequency-restricted reduced-order model to capture the input-output behaviour of the original system over the most restricted frequency range $\omega \in[11,14]$. We define the quantity

$$
e_{H[11,14]}=\frac{\|\hat{H}(\omega)-H(\omega)\|_{2}^{\omega \in[11,14]}}{\|H(\omega)\|_{2}^{\omega \in[11,14]}}
$$

and plot it as a function of the number of included modes (see figure $11 a$ ). In the above expression $\hat{H}(\omega)$ stands for the transfer function corresponding to the four cases. For all cases, the error decreases as more modes are added to the reduced-order model. As expected, the model corresponding to case 4 (blue curve) best approximates the input-output behaviour of the original system; the number of modes to do so is significantly lower than for the reference case.

For the closed-loop measure we take (similarly to the previous section) the real part of the least stable eigenvalue of the fully compensated system. This quantity 

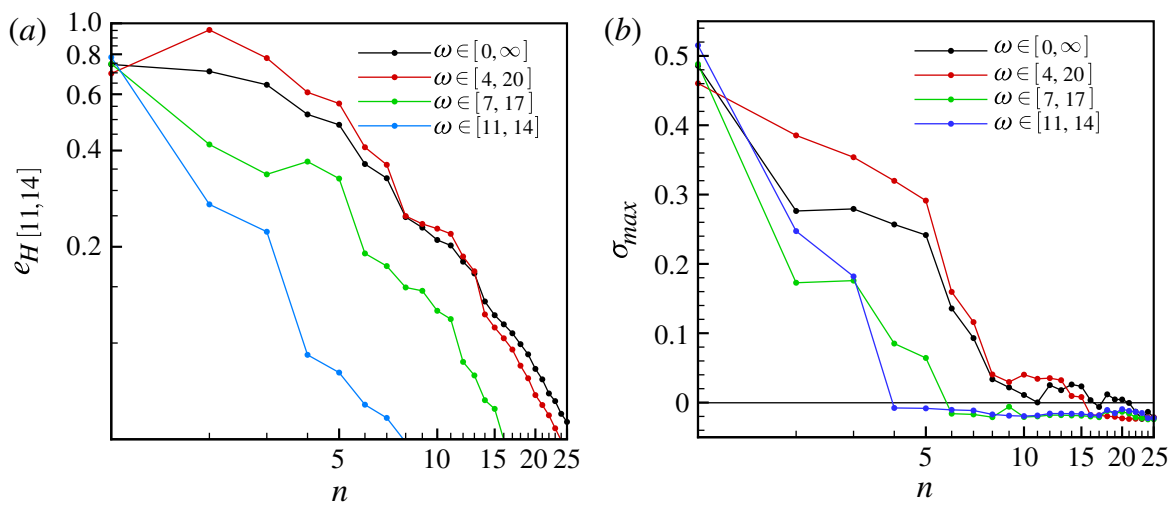

FIGURE 11. (a) Relative error of the transfer function versus number of modes for reducedorder models based on frequency-restricted POD modes. (b) Maximum growth rate of the fully compensated system based on frequency-restricted reduced-order models.

is displayed in figure 11(b) versus the number of modes included in the reducedorder model. Again, increasing the number of modes in the reduced-order model improves the stability of the compensated system. Significant differences, however, are discernible as the frequency range is increasingly restricted. While for the standard (unfiltered) POD-based model, 21 modes are necessary to stabilize the flow, only 16, 6 and 4 modes (for cases 2, 3 and 4, respectively) are needed to achieve a stable compensated configuration. This result further supports the fact that only the input-output behaviour of the most unstable modes is relevant for an effective reducedorder control performance.

\section{Reynolds number dependence}

It is apparent from the previous sections that the performance of reduced-order models based on global modes depends on the details of the instabilities that have to be controlled, which in turn depend on the Reynolds number. The goal of this section is to explore the range of Reynolds numbers for which closed-loop control can be successfully applied with a compensator based on global modes.

\subsection{Changes in the spectrum}

We start by studying how the stability properties of the uncontrolled flow are modified as the Reynolds number $R e$ is increased. In figure 12 the spectrum (in the $\omega>0$ half-plane) is displayed for Reynolds numbers from $R e=4800$ to 7500 . Only the branch containing the unstable global modes and the least stable of the stable branches are shown. The general behaviour is a destabilization of the spectrum as the Reynolds number increases. The unstable branch is further displaced into the unstable half-plane and the number of unstable modes increases. The stable eigenvalues move closer to the unstable half-plane, but ultimately remain stable. This behaviour is to be expected from an advection-diffusion problem as the diffusive terms play an increasingly minor role. While the decay rates are affected noticeably, the frequencies and spatial shapes (not shown) seem rather insensitive to Reynolds number variations. 


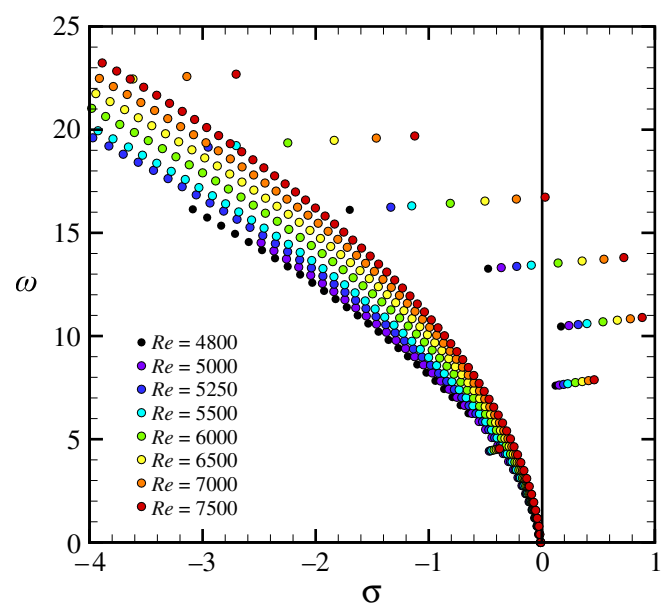

FIGURE 12. Changes in the global spectrum of flow over an open cavity as the Reynolds number is varied from $R e=4800$ to $R e=7500$.

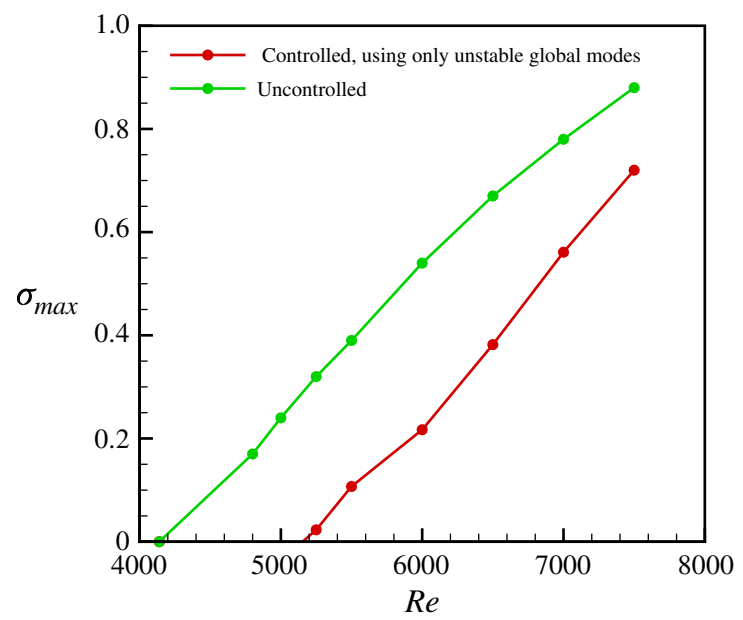

FIGURE 13. Growth rate of the least stable eigenvalue of the (green) uncontrolled and the (red) compensated system using a reduced-order model based on the unstable global modes only.

\subsection{Changes in the stability of the controlled system}

An interesting question concerns the ability of the compensator to stabilize the flow for a range of Reynolds numbers. To this end, a compensator based on global modes of the flow at a given Reynolds number is computed, after which the previously described method will be employed to determine the least stable eigenvalue of the compensated problem - and thus the performance of the reduced-order control problem.

\subsubsection{Compensators based only on the unstable global modes}

Previously it was argued that the unstable global modes of the flow have to be accounted for in the reduced-order model. The stable subspace, though, is not necessarily required, which prompts the questions (i) whether the flow can 

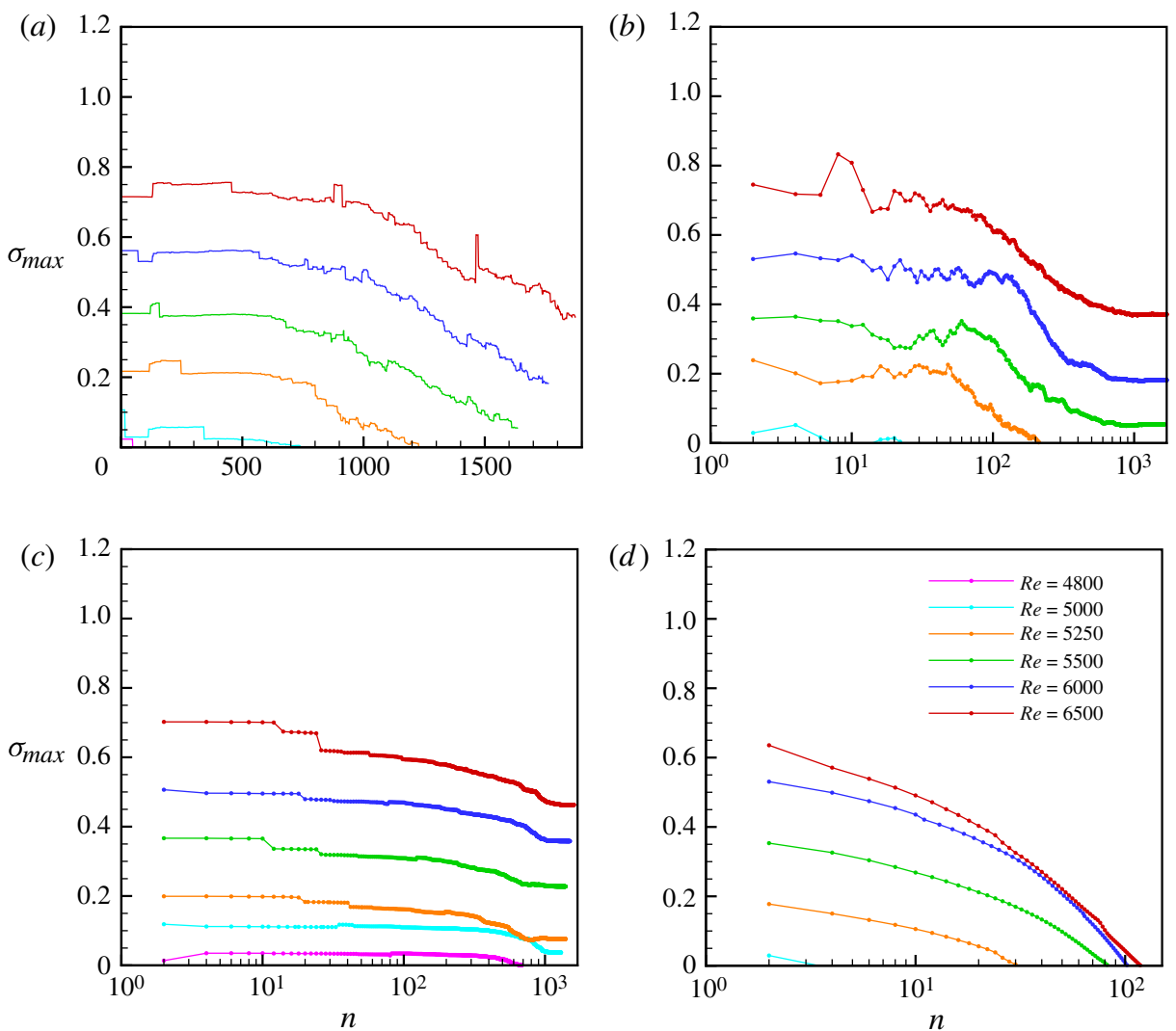

FIGURE 14. Growth rate of the least stable eigenvalue of the compensated system as global modes are added to the reduction basis according to the four selection criteria (see text): (a) growth rate, $(b)$ contribution to the input-output behaviour $\Gamma_{i},(c)$ orthogonal projections $\tilde{\Gamma}_{i}$, and $(d)$ 'quasi-optimal' stability ranking. The Reynolds numbers range from $R e=4800$ to $R e=6500$. Stabilization of the compensated system is achieved when $\sigma_{\max }<0$.

be stabilized using only the unstable modes and (ii) if so, up to what Reynolds number such a primitive compensator will be successful. In answer to these questions, figure 13 displays the growth rate of the least stable mode $\left(\sigma_{\max }\right)$ of the closedloop system where the compensator consists of only the unstable global modes. For Reynolds numbers less than $R e \approx 5250$, the growth rate $\sigma_{\max }$ is indeed negative which indicates that the flow can be stabilized without accounting for the stable subspace in the reduced-order model. For Reynolds numbers above $R e \approx 5250$, the instability grows too strong, and reduced-order models based on merely the unstable global modes cease to succeed in stabilizing the flow.

In Barbagallo et al. (2009) it has been shown that the flow can be stabilized at $R e=7500$, if relevant information about the stable subspace, in this case using proper orthogonal decomposition (POD) modes or balanced (BPOD) modes, is incorporated into the reduced-order model. The next section explores the question of whether the same can be accomplished by including global modes where Reynolds numbers ranging from $R e=5250$ to $R e=7500$ are considered. 


\subsubsection{Compensators based on the unstable and selected stable global modes}

Unstable and stable global modes are added to the reduction basis $V$ to obtain a reduced-order model of the flow. Stable modes are included according to one of the four ranking criteria defined in $\S 4.3$. In figure 14 the largest growth rate $\sigma_{\max }$ of the compensated system is displayed. Each subplot represents a specific ranking criterion; the various colours denote different Reynolds numbers.

In figure 14(a), stable global modes are included in the ROM according to their damping rate. Independent of the Reynolds number, the curves display similar behaviour. As the first stable modes are included, each curve shows a nearly constant plateau, until approximately $n=500$ stable global modes have been added; at this point, $\sigma_{\max }$ decreases as more modes are incorporated into the reduced-order model. If the initial instability is sufficiently weak, as is the case for $R e=5250,5500$ and 6000 , the system can eventually be stabilized. However, even with all global modes (up to $\sigma>-4$ ) included, flow over an open cavity for Reynolds numbers larger than $R e \approx 6500$ can no longer be rendered stable by a compensator based on global modes for our choice of LQG control parameters.

When the global modes are ranked according the $\Gamma$-criterion (see figure 14b) analogous conclusions can be drawn. However, similarly to the case $R e=7500$ studied in $\S 5$, the stabilization of the compensated system occurs at a lower number of stable modes. In figure $14(c)$ we consider stable global modes ranked by the $\tilde{\Gamma}$-criterion. In this case, the system can only be stabilized for $R e=4800$. As mentioned previously, the criterion does not yield favourable results for our case, even though it has been successfully applied to other configurations (Ehrenstein et al. 2010).

In contrast (and as expected), the system can eventually be stabilized for all considered Reynolds numbers $(5250 \leqslant R e \leqslant 7500)$ if the 'quasi-optimal' ranking is employed (see figure 14d).

The above results demonstrate that some unstable flows can be stabilized using reduced-order models based on global modes. Despite that, the number of stable global modes necessary to model the stable subspace dynamics increases dramatically with Reynolds number. The question then arises of whether, given an arbitrary (high) Reynolds number, a sufficient number of stable global modes can be computed accurately so that such a reduced-order model can be constructed. In our case, a large but still finite number of global modes could be calculated, before round-off error deteriorated the iterative computations. This phenomenon can be linked to the nonnormality of the linearized Navier-Stokes matrix $A$ which makes iterative eigenvalue computations an ill-conditioned (and ill-fated) undertaking. But as more stable global modes become necessary to stabilize the flow at larger Reynolds numbers, the finite limit of computable modes will at last be reached.

Another relevant question concerns the reason for the drastic increase in stable global modes needed to stabilize the flow at higher Reynolds numbers. A facile and simplistic answer would argue that the stable subspace is generally less damped (see $\S 6.1$ ) which generates a more complex dynamics and, in turn, a larger number of degrees of freedom. To follow up this argument, we consider a compensator based on the unstable global modes and POD modes to model the stable subspace. The minimum number of POD modes required to stabilize the flow is shown in figure 15 . The flow can be stabilized at each Reynolds number considered using at most thirty POD modes to represent the stable subspace dynamics. Clearly, this behaviour runs counter to the claim that the complexity of the stable subspace requires a reducedorder model of high dimensions. We are left with the fact that a few thousand stable global modes need to be computed, ranked and incorporated into a reduced-order 


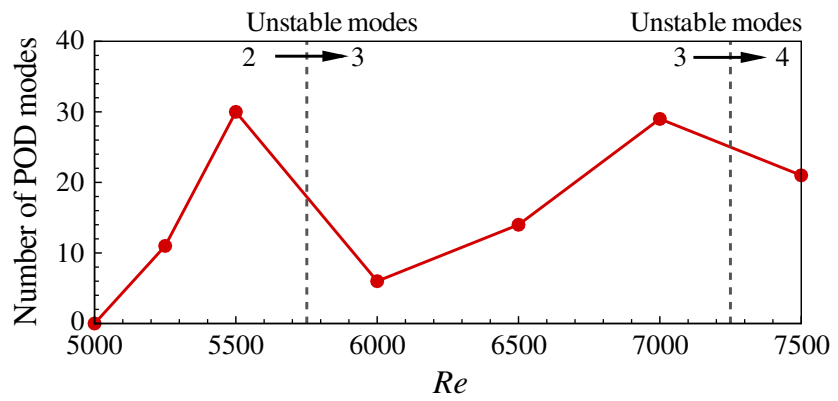

FIGURE 15. (Colour online) Minimum number of proper orthogonal decomposition (POD) modes to stabilize the compensated system consisting of a reduced model based on unstable global modes and POD modes. At critical values of the Reynolds number (indicated by dashed lines) additional global modes become unstable.

model in order to represent a subspace dynamics that can equally well (or better) be described by thirty POD modes; we thus conclude that global modes constitute a poor basis when designing closed-loop control strategies using reduced-order models. Our study suggests that a reduced-order model based on global modes is capable of stabilizing systems that are only weakly unstable (such as the configurations studied by Åkervik et al. 2007 and Ehrenstein et al. 2010). If stronger instabilities (such as the one studied in Barbagallo et al. 2009) are encountered, reduced-order models based on global modes will fail and different reduction bases, such as POD modes or balanced modes, have to be explored.

\section{Summary and conclusions}

Projection-based model reduction techniques leave a great many choices to compute low-dimensional systems from high-dimensional models. In this article, we have investigated the suitability of global modes in closed-loop control applications of oscillator-type flows (in particular, the flow over an open cavity at supercritical Reynolds numbers).

The reduced-order models are composed of unstable global modes, capturing the inherent instability, and selected stable global modes, representing the stable subspace of the perturbation dynamics. The selection of stable global modes is critical and has been carried out based on four criteria, yielding four different reduced-order models composed of global modes. Using a representative test case $(R e=7500)$ the open-loop behaviour of these models has first been assessed, which revealed that the damping rate (criterion 1, Åkervik et al. 2007) is a poor indicator in selecting global modes, while choosing global modes with high controllability and observability but low damping rate (criterion 2, Bagheri et al. 2009a; Barbagallo et al. 2009) showed better performance but nonetheless ultimately failed. A selection criterion (criterion 3, Ehrenstein et al. 2010) based on double-projection could not demonstrate the same advantages and potential as reported in Ehrenstein et al. (2010), but rather showed disappointing convergence behaviour when applied to our configuration. A fourth criterion, an a posteriori-selection rule, has been added both as a benchmark to gauge near-optimal results and as verification that highly damped modes have to be considered to capture the correct input-output behaviour. The closed-loop performance of the different models has been characterized by the stability of the compensated system. With the exception of the artificial fourth criterion ('quasi-optimal' stability 
ranking), all computed systems were found unstable for the test case, thus confirming the conclusions drawn from the open-loop study.

While the unstable global modes in the reduction basis capture the part of the flow dynamics representing the overall oscillatory behaviour, the stable global modes are ineffective in describing the remaining behaviour. This latter flow behaviour is akin to an amplifier, and the global modes do not appear to form a proper basis to describe this type of behaviour. Physically, amplifier flows are dominated by a convective disturbance dynamics which is known to be poorly represented by global modes.

An interesting finding of our analysis was the strong evidence that the commonly applied $\mathscr{H}_{2}$ - and $\mathscr{H}_{\infty}$-measures are not optimal to evaluate the open-loop behaviour of reduced-order models and their performance in feedback control applications, a fact that is commonly known in the field of robust control. Since global instabilities occur at discrete frequencies, the compensator responds at these same frequencies. For this reason, it seems sensible that a reduced-order compensator that captures the perturbation dynamics in the vicinity of these frequencies well should perform better - or more efficiently - than a compensator that approximates the transfer function over a wide range of (some dynamically irrelevant) frequencies or puts emphasis on minimizing the largest-magnitude error with no concern at which frequency this maximum error occurs. This reasoning is in contrast to the widely accepted $\mathscr{H}_{\infty}$-norm minimization intrinsic to balanced truncation (see Antoulas 2005) and may warrant a re-evaluation of proper input-output measures that favour physically motivated frequency ranges over mathematically inspired optimization. Based on our observations, a frequency-restricted $\mathscr{H}_{2}^{\omega}$-norm, which accurately links open-loop and closed-loop performance analysis, has been proposed and defined. Applications and consequences of this definition will be further explored in a future work.

The limits of stabilizability using reduced-order models based on global modes have been quantified by conducting a parameter study in the Reynolds number ranging from the critical value of $R e_{c}=4140$ to $R e=7500$. For very weak global instabilities $(R e \leqslant 5250)$ only the unstable modes suffice to stabilize the system. Thus, even entirely neglecting the stable subspace dynamics, the LQG compensator successfully suppressed the global instability. This seeming robustness of the compensator, however, does not extend far beyond weak instabilities: already moderate instabilities can no longer be controlled, even if stable global modes are added. The flow cases considered in Åkervik et al. (2007) and Ehrenstein et al. (2010) are believed to fall into the 'weak' category where reduced-order compensators accounting for all unstable and a few stable modes are still able to stabilize the flow.

Nevertheless, considering the limitation of controlling only weak instabilities, recognizing the difficulty of computing a large number of global modes owing to nonnormality and acknowledging the lack of a rigorous and effective selection criterion, it must be concluded that global modes usually do not constitute a suitable choice of reduction basis for closed-loop control applications. A hybrid approach, with unstable global modes capturing the oscillatory flow behaviour and e.g. POD modes describing the convective flow behaviour, represents a far more promising approach to model reduction and control design. 

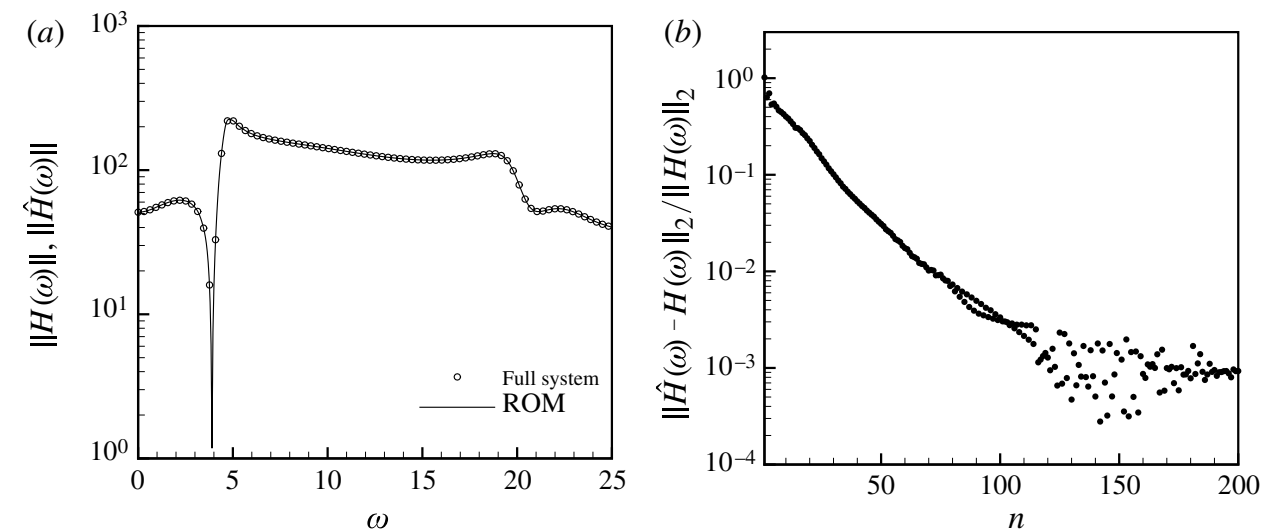

FIGURE 16. (a) Magnitude of the transfer function of the full system (symbols) and the reduced-order mode based on POD modes (solid line). (b) Relative $\mathscr{H}_{2}$-error of the transfer function of the reduced-order plant model based on $n$ POD modes.

\section{Appendix. Replacing the full system by a reduced-order model}

The behaviour of an unstable system can be separated into its unstable and stable dynamics, which decouple. The system matrix $A$ can be decomposed according to

$$
A=\left(\begin{array}{cc}
A_{u} & 0 \\
0 & A_{s}
\end{array}\right)
$$

where $A_{u}$ (resp. $A_{s}$ ) denotes the matrix governing the unstable (resp. stable) states (see Barbagallo et al. 2009; Ahuja \& Rowley 2010). We wish to replace the full system by a reduced model which (i) still captures the unstable dynamics of the original system and (ii) accurately reproduces the input-output behaviour, that is, the link between actuator and sensor. The first requirement is satisfied by choosing the unstable global modes for a reduction basis. For the Reynolds numbers considered in this work the dimension of the unstable subspace (dimension of $A_{u}$ ) varies from two to eight. For the second requirement we choose proper orthogonal decomposition (POD) modes to express the stable subspace dynamics. This choice has been shown (see e.g. Barbagallo et al. 2009) to result in an accurate description of the stable input-output behaviour with a moderate number of modes. The POD modes are computed for each Reynolds number using a snapshot method (Sirovich 1987). An impulse response is computed based on (5.1) until a dimensionless time of $T=20$ resulting typically in a decrease of the perturbation energy by three orders of magnitude. Snapshots are extracted from the linearized simulations at equi-spaced time intervals of $\Delta t=0.02$. The POD modes are then computed from these snapshots and used in a Petrov-Galerkin projection of the system (2.7) resulting in

$$
\begin{gathered}
\frac{\mathrm{d} \hat{\boldsymbol{X}}}{\mathrm{d} t}=\hat{A}_{s}^{p o d} \hat{\boldsymbol{X}}+\hat{\boldsymbol{B}}_{s}^{p o d} u, \\
m=\hat{\boldsymbol{C}}^{\text {pod }} \hat{\boldsymbol{X}},
\end{gathered}
$$

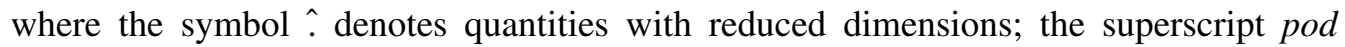
indicates a reduction based on POD modes. 
The transfer function of the reduced-order model is given by $\hat{H}(\omega)=\hat{\boldsymbol{C}}^{\text {pod }}(\mathrm{i} \omega /-$ $\left.\hat{A}_{s}^{\text {pod }}\right)^{-1} \hat{\boldsymbol{B}}_{s}^{\text {pod }}$ and is displayed for $R e=7500$ using 150 POD modes in figure 16(a) by a solid line together with the transfer function of the full system (in symbols). Very good agreement is observed which is confirmed by computing the relative error between the reduced-order and full transfer functions using the $\mathscr{H}_{2^{-}}$and $\mathscr{H}_{\infty^{-}}$ norm. Results are shown in figure $16(b)$; both curves decrease rapidly and eventually converge to an acceptable error for about 120 POD modes. Similar results have been obtained at lower Reynolds numbers (not shown here); thus, reduced-order models for the full linearized dynamics based on 150 POD modes will be considered for each Reynolds number. It is important to keep in mind, however, that the reduced-order model does not capture the complete dynamics of the stable subspace but only the part relevant to our study, namely the input-output behaviour between actuation and measurement.

In summary, the system given by (2.7) will be replaced by the reduced-order model

$$
\begin{aligned}
\frac{\mathrm{d}}{\mathrm{d} t}\left(\begin{array}{l}
\hat{\boldsymbol{X}}_{u}^{g m} \\
\hat{\boldsymbol{X}}_{s}^{\text {pod }}
\end{array}\right) & =\left(\begin{array}{cc}
\hat{\boldsymbol{\Lambda}}_{u} & 0 \\
0 & \hat{A}_{s}^{\text {pod }}
\end{array}\right)\left(\begin{array}{l}
\hat{\boldsymbol{X}}_{u}^{g m} \\
\hat{\boldsymbol{X}}_{s}^{\text {pod }}
\end{array}\right)+\left(\begin{array}{l}
\hat{\boldsymbol{B}}_{u}^{\text {gm }} \\
\hat{\boldsymbol{B}}_{s}^{\text {pod }}
\end{array}\right) u, \\
m & =\left(\begin{array}{ll}
\hat{\boldsymbol{C}}_{u}^{\text {gm }} & \hat{\boldsymbol{C}}_{s}^{\text {pod }}
\end{array}\right)\left(\begin{array}{l}
\hat{\boldsymbol{X}}_{u}^{g m} \\
\hat{\boldsymbol{X}}_{s}^{\text {pod }}
\end{array}\right),
\end{aligned}
$$

which is based on unstable global modes for representing the unstable behaviour (indicated by the superscript $\mathrm{gm}$ ) and on POD modes for capturing the input-output dynamics contained in the stable subspace (indicated by the superscript pod).

\section{REFERENCES}

Ahuja, S. \& Rowley, C. W. 2010 Feedback control of unstable steady states of flow past a flat plate using reduced-order estimators. J. Fluid Mech. 645, 447-478.

Åkervik, E., Hepffner, J., Ehrenstein, U. \& Henningson, D. S. 2007 Optimal growth, model reduction and control in a separated boundary-layer flow using global modes. J. Fluid Mech. 579, 305-314.

Antoulas, A. C. 2005 Approximation of Large-Scale Dynamical Systems. SIAM.

Antoulas, A., Sorensen, D. \& Gugercin, S. 2001 A survey of model reduction methods for large-scale systems. Contemp. Maths 280, 193-219.

BAgheri, S., BRANDT, L. \& Henningson, D. S. $2009 a$ Input-output analysis, model reduction and control of the flat-plate boundary layer. J. Fluid Mech. 620, 263-298.

Bagheri, S., Heepffner, J., Schmid, P. J. \& Henningson, D. S. $2009 b$ Input-output analysis and control design applied to a linear model of spatially developing flows. Appl. Mech. Rev. 62 (2), 020803.

Barbagallo, A., Sipp, D. \& Schmid, P. J. 2009 Closed-loop control of an open cavity flow using reduced-order models. J. Fluid Mech. 641, 1-50.

Berkooz, G., Holmes, P. \& Lumley, J. L. 1993 The proper orthogonal decomposition in the analysis of turbulent flows. Annu. Rev. Fluid Mech. 25, 539-575.

BEWLEY, T. R. \& LiU, S. 1998 Optimal and robust control and estimation of linear paths to transition. J. Fluid Mech. 365, 305-349.

Burl, J. B. 1999 Linear Optimal Control. $\mathrm{H}_{2}$ and $\mathrm{H}_{\infty}$ Methods. Addison-Wesley.

DAtTA, B. 2003 Numerical Methods for Linear Control Systems. Academic.

Dergham, G., SipP, D., Robinet, J.-C. \& Barbagallo, A. 2011 Model reduction for fluids using frequential snapshots. Phys. Fluids 23, 064101. 
Ehrenstein, U., Passaggia, P.-Y. \& Gallaire, F. 2010 Control of a separated boundary layer: reduced-order modeling using global modes revisited. Theor. Comput. Fluid Dyn., 25 (1), 195-207.

Giannetti, F. \& LUChini, P. 2007 Structural sensitivity of the cylinder wake's first instability. J. Fluid Mech. 581, 167-197.

Hecht, F., Pironneau, O., Hyaric, A. L. \& Ohtsuka, K. 2005 Freefem + +, The Book. UPMC-LJLL.

Henningson, D. S. \& ÅKervik, E. 2008 The use of global modes to understand transition and perform flow control. Phys. Fluids 20, 031302.

Kim, J. \& Bewley, T. R. 2007 A linear systems approach to flow control. Ann. Rev. Fluid Mech. 39, 383-417.

LehoucQ, R. B. \& ScotT, J. A. 1997 Implicitly restarted Arnoldi methods and subspace iteration. SIAM J. Matrix Anal. Applics. 23, 551-562.

LuMley, J. L. 1970 Stochastic Tools in Turbulence. Academic.

MARquet, O., SiPP, D. \& JACQUin, L. 2008 Sensitivity analysis and passive control of cylinder flow. J. Fluid Mech. 615, 221-252.

MoORE, B. 1981 Principal component analysis in linear systems: controllability, observability, and model reduction. IEEE Trans. Autom. Control 26, 17-32.

Rowley, C. W. 2005 Model reduction for fluids using balanced proper orthogonal decomposition. Intl J. Bifurcation Chaos 15, 997-1013.

Schmid, P. J. \& Henningson, D. S. 2000 Stability and Transition in Shear Flows. Springer.

SiPP, D. \& LEBEDEV, A. 2007 Global stability of base and mean flows: a general approach and its applications to cylinder and open cavity flows. J. Fluid Mech. 593, 333-358.

Sipp, D., Marquet, O., Meliga, P. \& Barbagallo, A. 2010 Dynamics and control of global instabilities in open flows: a linearized approach. Appl. Mech. Rev. 63, 030801.

Sirovich, L. 1987 Turbulence and the dynamics of coherent structures. Q. Appl. Maths 45, 561-590.

Strykovski, P. J. \& SReenivasan, K. R. 1990 On the formation and suppression of vortex shedding at low Reynolds numbers. J. Fluid Mech. 218, 71-107.

Willcox, K. \& Peraire, J. 2002 Balanced model reduction via proper orthogonal decomposition. AIAA J. 40, 2323-2330.

Zhou, K., Doyle, J. C. \& Glover, K. 2002 Robust and Optimal Control. Prentice Hall. 